\title{
In-situ-grown silver-polymer framework with coordination complexes for functional artificial tissues
}

Swee Ching Tan ( $\nabla$ msetansc@nus.edu.sg )

National University of Singapore https://orcid.org/0000-0003-2074-8385

Songlin Zhang

National University of Singapore

Yibing Deng

Nanjing University

Alberto Libanori

University of California, Los Angeles

Yihao Zhou

University of California, Los Angeles

Jiachen Yang

National University of Singapore

Trinny Tat

University of California, Los Angeles

Lin Yang

National University of Singapore

Wanxin Sun

Bruker

Peng Zheng

Nanjing University

Youliang Zhu

Jilin University

Jun Chen

University of California, Los Angeles https://orcid.org/0000-0002-3439-0495

\section{Article}

Keywords: sensorized actuators, neuromuscular systems, silver-polymer framework

Posted Date: October 12th, 2021

DOI: https://doi.org/10.21203/rs.3.rs-843180/v1 
License: (c) (i) This work is licensed under a Creative Commons Attribution 4.0 International License. Read Full License 
In-situ-grown silver-polymer framework with coordination complexes for functional artificial tissues

Songlin Zhang ${ }^{1}$, Yibing Deng ${ }^{2}$, Alberto Libanori ${ }^{3}$, Yihao Zhou ${ }^{3}$, Jiachen Yang ${ }^{1}$, Trinny Tat ${ }^{3}$, Lin Yang ${ }^{1}$, Wanxin Sun ${ }^{4}$, Peng Zheng ${ }^{2}$, You-Liang Zhu ${ }^{5 *}$, Jun Chen ${ }^{3 *}$, Swee Ching Tan ${ }^{1 *}$

${ }^{1}$ Department of Materials Science and Engineering, National University of Singapore, 9 Engineering Drive 1, Singapore, 117574, Singapore

${ }^{2}$ State Key Laboratory of Coordination Chemistry, Chemistry and Biomedicine Innovation Center (ChemBiC), School of Chemistry and Chemical Engineering, Nanjing University, Nanjing, Jiangsu, 210023, P.R. China

${ }^{3}$ Department of Bioengineering, University of California, Los Angeles, Los Angeles, CA 90095, USA

${ }^{4}$ Bruker Nano Surface and Metrology, 30 Biopolis Street \#09-01, The Matrix, Singapore 138671

${ }^{5}$ State Key Laboratory of Supramolecular Structure and Materials, College of Chemistry, Jilin University, Changchun 130012, China

"To whom correspondence should be addressed: Y.-L.Z. (youliangzhu@jlu.edu.cn), S.W.T (msetansc@ nus.edu.sg), J.C. (jun.chen@ucla.edu)

Sensorized actuators are critical to imitate proprio-/exteroception properties of biological neuromuscular systems. Existing add-on approaches, which physically blend heterogeneous sensor/actuator components, fall short of yielding satisfactory solutions, considering their suboptimal interfaces, poor adhesion, and electronic/mechanical property mismatch. Here, we report a single homogeneous material comprising seamless sensing-actuation unification properties at nano-/molecule levels, in which built-in sensing functions originate from the actuator architecture itself. In-situ-grown silver nanoparticles and metal-ligand complexes cooperatively create a silver-polymer framework (SPF) that is stretchable (1200\%), conductive $(0.076 \mathrm{~S} / \mathrm{m})$, and strong (0.76 MPa in-strength). SPF displays concomitant multimodal sensing (mechanical and thermal cues) and sensorized actuation capabilities, which include proprio-deformation and external stimuli perceptions (simultaneous with load-lifting ability up to $3700 \times$ of own weight). In view of its human somatosensitive muscular systems imitative functionality, the reported SPFs bode well for use with next generation functional tissues including artificial skins, human-machine interfaces, selfsensing robots, and otherwise dynamic materials. 
Muscles are admired for their remarkable ability to sense and interact with the external environment simultaneously via their inherent structure - an integrated system with receptors and motor structures evenly distributed throughout a biological network (Fig. 1a) ${ }^{1}$. Such natural models inspire research avenues aimed at recreating coupled somatosensory and motor functions using synthetic substances ${ }^{2-5}$, where sensation and actuation are impeccably fused into a single stand-alone, dynamic material platform. This functions-unified system is expected to play a crucial role as an alternative platform to help exploit biological somatosensitive muscular system functionalities ${ }^{6,7}$. Efforts in devising artificial sensorimotor abilities ex novo, have focused on the use of soft and stretchable materials in light of their incomparable transmutability, conformability, and design freedom, especially when compared to rigid counterparts (i.e., conventional sensors or actuators $)^{8-10}$. Although notable advances on soft sensors and actuators have been reported individually $^{11-13}$, their distinct working mechanisms (i.e., triboelectricity, piezoresistivity, piezoelectricity or colorimetry for sensing; and hydraulics, pneumatics, light, heat or electricity for actuation) have largely impeded a seamless sensing-actuation integration within a single material unit. For instance, the physical blending of various functional components to unify sensing-actuation as exemplified in conventional add-on approaches has shown interfacing problems, coupled with issues of multi-step integration, stress concentrations, poor adhesions, as well as the presence of dense wiring ${ }^{14-18}$. The resulting electronic/mechanical property mismatch among varying materials poses a dilemma to achieving the trinity of desirable properties, notably: sensing, actuation, and ideal integration of various functional parts. Thus, developing a stand-alone and dynamic-material-based platform for simultaneous sensory perception and stimulusresponsive motility (self-sensing actuator), which is established on a homogenous system where heterogeneous sensing and actuation functions are mutually assembled into a single material unit, remains an unmet goal.

One promising approach in devising muscle-like intelligent structures with ideal sensing-actuation unification is to use built-in techniques, by which sensing abilities originate from the actuator architecture itself ${ }^{19}$. However, current methods which rest on percolation theory or on the use of specific device structure design still suffer from the poor dispersion quality and interface compliance among external conductive fillers (for sensing) and substrate materials (for actuating) (Fig. 1b), as well as complexity on actuators' structural (geometry) optimization, which limits the 
sensing modality ${ }^{20,21}$. Even though improvements for soft and stretchable self-sensing actuators, primarily using (ionic or electronic) conductive hydrogel composites with organic or inorganic fillers, have been made ${ }^{22}$, issues of ion leakage for unstable sensing and heterogeneity of filler dispersion for deteriorated mechanical deformation ability, still limit the fabrication of superlative sensing-actuation integration within a single material system. Furthermore, enabling multimodal sensory perceptions would, discouragingly, further complicate fabrication approaches ${ }^{12}$. Consequently, a simple and effective processing technique is particularly necessary to help construct somatosensitive actuators in a single homogeneous artificial material platform, which must ultimately be soft, stretchable, and self-sensing (proprio-/exteroception) with muscle-like actuation performance.

We developed a single homogeneous material platform silver-polymer framework (SPF) with simultaneous sensing and actuation capabilities at nano- and molecule-level unification, using a one-step process to help mimic the somatosensitive muscular system. Specifically, the SPF was developed by leveraging on the basis of mechanical deformation functionality from dynamic $\left[\mathrm{Ag}(-\mathrm{C} \equiv \mathrm{N})_{x}\right]^{+}$complexes (for actuating) and electronic functionality from in-situ-grown silver nanoparticles (AgNPs, for sensing). The SPF was constructed by combining polyacrylonitrile (PAN) and silver ion (from $\mathrm{AgNO}_{3}$ ) in a reducing solvent environment of dimethylformamide (DMF) (hereafter denoted as PANSion). The obtained PANSion was shown to be conductive $\left(0.076 \mathrm{~S} \cdot \mathrm{m}^{-1}\right)$, soft/stretchable (1200\% strain), yet strong (>3700× own-weight lifting capability, surpassing most of the state-of-the-arts and human muscles) (Supplementary Table 1). These properties, which originate from the one-step process formation of $\left[\mathrm{Ag}(-\mathrm{C} \equiv \mathrm{N})_{x}\right]^{+}$complexes with concomitant in-situ-grown AgNPs (Fig. 1c), enable multimodal sensing ability and seamless sensing-actuation unification, providing desired and previously unattained self-sensing actuation capabilities in a single homogenous material unit (Extended Data Fig. 1). In particular, we highlight the PANSion's application versatility by exploring its use in four representative applications: a multistimuli-responsive PANSion e-skin (responsive to mechanical and thermal cues), a sensing fabric via dip-coating PANSion solution, a PANSion fiber via in-air spinning, and a self-sensing PANSion-based pneumatic actuator. This work elucidates the broad applicability of PANSion in imitating natural tissues' versatile functions using a single homogeneous material platform, paving the way towards more integrated wearable devices and life-like soft robotics with 
multimodal sensing and self-sensing actuation abilities, much like the biological somatosensitive muscular system.

\section{Design and characterization of $\left[\mathrm{Ag}(-\mathrm{C} \equiv \mathrm{N})_{x}\right]^{+}$coordination complexes}

Using PAN as a starting material, we first constructed metal-ligand complexes of $\left[\mathrm{Ag}(-\mathrm{C} \equiv \mathrm{N})_{x}\right]^{+}$ among PAN chains by adding silver nitrate (see Methods), yielding an entangled PANSion chain network that is critical to forming a stretchable system. Compared with a PAN solution, which exhibits molecular interactions of $-\mathrm{C} \equiv \mathrm{N}$ vs. $-\mathrm{N}-\mathrm{C}=\mathrm{O}(\mathrm{PAN} v s . \mathrm{DMF})$, and $-\mathrm{C} \equiv \mathrm{N}$ vs. $-\mathrm{C} \equiv \mathrm{N}(\mathrm{PAN}$ vs. PAN), a PANSion solution ought to have an additional interaction from $\left[\mathrm{Ag}(-\mathrm{C} \equiv \mathrm{N})_{x}\right]^{+}$ complexes (Fig. 1d right). Given this, we verified the chemical environment change of $-\mathrm{C} \equiv \mathrm{N}$ using Fourier-transform infrared (FTIR) spectroscopy, which indicated constrained bond stretching with decreased intensity (Fig. 1d left; and Supplementary Fig. 1) due to the formation of $\left[\mathrm{Ag}(-\mathrm{C} \equiv \mathrm{N})_{x}\right]^{+}$complexes $^{23}$. Additionally, $\left[\mathrm{Ag}(-\mathrm{C} \equiv \mathrm{N})_{x}\right]^{+}$complexes also induce a structural change from an original semicrystalline PAN to an amorphous PANSion (Supplementary Fig. 2). Based on the density functional theory (DFT) calculations (Supplementary Text 1), we inferred that the binding energy between the metal ion and $-\mathrm{C} \equiv \mathrm{N}$ ligand was vital to obtaining a soft and stretchable network, considering only the PANSion (i.e., $\operatorname{Ag}(\mathrm{I}), \sim 134.55 \mathrm{~kJ} \cdot \mathrm{mol}^{-1}$ ) sample showed excellent stretchability compared to the other films (Extended Data Fig. 2), which were prepared using several different metal ions with high binding energy (Supplementary Table 2, and Supplementary Figs. 3-4).

To originate and confirm the stretchability of PANSion networks, we characterized mechanical properties of $\left[\mathrm{Ag}(-\mathrm{C} \equiv \mathrm{N})_{x}\right]^{+}$complexes at single-molecule level, using atomic force microscopy (AFM)-based single-molecule force spectroscopy (SMFS, see Methods) ${ }^{24}$. Stretching PANSion by AFM-SMFS resulted in a force-extension curve with a clear detachment force peak, which could be assigned to the dissociation of $\left[\operatorname{Ag}(-\mathrm{C} \equiv \mathrm{N})_{x}\right]^{+}$complexes (either intra- or inter-chain mechanical failures as indicated by three individual curves in Fig. 1e, colored in purple/green/blue). By contrast, the control experiment on PAN chains recorded no distinguishable signals, possibly due to the molecular chains' free movement in DMF solvent (Fig. 1e, black line). Additionally, the contour length, $L_{\mathrm{c}}$ (length detected right before the force rupture peaks), was 
observed to vary from 106 to $389 \mathrm{~nm}$ (Extended Data Fig. 3a-e), which suggested random formation sites of coordination complexes formation along the molecule chains. These results validated that $\left[\operatorname{Ag}(-\mathrm{C} \equiv \mathrm{N})_{x}\right]^{+}$complexes, as cross-linkers, interconnected PAN molecules into a PANSion chain, exhibiting an extended effective-molecule-length (EML) (Supplementary Text 2). Notably, the cyclic stretching-releasing cycle test done via AFM-SMFS showed that the PANSion chains are highly elastic (Fig. 1f). Taken together, the cross-linked PANSion chain network displayed excellent molecular stretchability, where mechanical ruptures of $[\mathrm{Ag}(-\mathrm{C} \equiv$ $\left.\mathrm{N})_{x}\right]^{+}$complexes could dissipate external energies during the stretching process. Note that $\left[\mathrm{Ag}(-\mathrm{C} \equiv \mathrm{N})_{x}\right]^{+}$complexes are also found to be dynamic, as based on binding energy calculations. However, we did not observe cyclic dissociation-reformation of $\left[\mathrm{Ag}(-\mathrm{C} \equiv \mathrm{N})_{x}\right]^{+}$complexes during AFM-SMFS tests, partially because PANSion's EML was still shorter than those of other reported polymer systems such as polydimethylsiloxane (Extended Data Fig. 3f-g) ${ }^{24}$.

\section{Rheological characterizations of PANSion solutions}

Upon confirming the stretchability of PANSion chains originating from $\left[\mathrm{Ag}(-\mathrm{C} \equiv \mathrm{N})_{x}\right]^{+}$complexes at single-molecule level, we examined the rheological properties of PANSion solutions at a macroscale to help elucidate the entanglement and elasticity of the polymer networks. The concentration of $\left[\operatorname{Ag}(-\mathrm{C} \equiv \mathrm{N})_{x}\right]^{+}$complexes, as cross-linkers, is key to fine tuning the polymer networks' entanglement and thus the PANSion's properties themselves. Consequently, we adjusted the weight ratio of $\mathrm{AgNO}_{3}: \mathrm{PAN}, \phi$, to investigate mechanical responses of five solutions $(\phi=0,0.5,1.0,1.5,2.0$, Supplementary Table 3) using rheological measurements (Supplementary Text 3). It is worth mentioning that gel transition for $\phi=2.0$ occurred after circa 10-days of storage at room temperature, although during this time, the other samples remained liquid (fig S8A), indicating that a physically/chemically entangled PANSion network $(\phi$ dependent) gradually forms as time passes by. To confirm this speculation, we examined the rheological properties of PANSion solutions (1-day storage), which presented an increased viscosity and modulus in concomitance with $\phi$ value increase from 0 to 2.0 (Fig. 1g, and Supplementary Fig. 5b-d). This could be ascribed to the increasingly cross-linkage of the network brought about by the $\left[\mathrm{Ag}(-\mathrm{C} \equiv \mathrm{N})_{x}\right]^{+}$complexes. Once the cross-linking density reached a threshold, gel transition occurred, resulting in viscosity of several magnitudes higher when 
compared to solution counterparts. Furthermore, the gelation evolution for $\phi=2.0$ was associated with the competing effect between storage modulus $\left(G^{\prime}\right)$ and loss modulus $\left(G^{\prime \prime}\right)$, where $G^{\prime}$ became dominant once gelation occurred (Supplementary Fig. 6), implying the presence of a strongly entangled and densely cross-linked PANSion network. These results are in accordance with other reports $^{25}$ and our AFM-SMFS data, affirming that $\left[\mathrm{Ag}(-\mathrm{C} \equiv \mathrm{N})_{x}\right]^{+}$complexes, as cross-linkers, cause structural changes to the PANSion network when compared to its antetype of PAN.

The dynamic mechanical properties of the samples were further studied after gelation (for $\phi=2.0$ ). Fig. 1h shows a broad linear gel region (orange lines) measured using strain-dependent oscillatory rheology, with a gel-to-sol cross-over point at $\sim 300 \%$. The high yield strain $(>100 \%)$ also confirmed the dynamic nature of $\left[\operatorname{Ag}(-\mathrm{C} \equiv \mathrm{N})_{x}\right]^{+}$complexes ${ }^{26}$. Fig. 1i shows the fast mechanical response with near full modulus recovery when alternating between 1 and 1000\% strain, implying excellent reversibility of PANSion gel due to the fast association kinetics of $\left[\mathrm{Ag}(-\mathrm{C} \equiv \mathrm{N})_{x}\right]^{+}$ complexes $^{25,27}$. Interestingly, we observed an increase of $G^{\prime}$ at high oscillation strain for $\phi=2.0$ when in a liquid-like gel state (i.e., flowable with a cross-over point >1200\%) before reaching solid-like state (green lines in Fig. 1h). Hypothetically, this could be reasoned to be the higher reformation rate of $\left[\mathrm{Ag}(-\mathrm{C} \equiv \mathrm{N})_{x}\right]^{+}$than its dissociation rate at a higher oscillation strain, a highly unusual observation. Thus, while experimental investigation on the dynamic nature of $[\mathrm{Ag}(-\mathrm{C} \equiv$ $\left.\mathrm{N})_{x}\right]^{+}$complexes by SMFS is one of our future research focus, we note that much work is to be done to consistently understand structural evolutions of the PANSion network from a solution to a liquid-like gel, and up to a fully solid gel state. On a separate note, the gel time of PANSion solutions was shown to be highly dependent on the $\phi$ value and the base concentration of the starting PAN solutions (Supplementary Fig. 7). Such a factor could be accelerated using thermal heating for various periods of time or at different temperatures (Fig. 1j and $\mathbf{k}$ ). This information helps establish the processing parameters for the preparation of freestanding PANSion films (hereafter, $\phi=2.0$ is used for further study unless otherwise specified).

\section{PANSion film with in-situ-grown AgNPs}

Having studied the properties of the PANSion molecule, solution, and gel, we were subsequently confident to prepare the stretchable and freestanding film, introducing $\left[\mathrm{Ag}(-\mathrm{C} \equiv \mathrm{N})_{x}\right]^{+}$complexes 
(Fig. 2a, and Supplementary Text 3) to do so. What substantially distinguished our PANSion from other coordination-complexes-based soft materials was indeed the concomitant implementation of conductive percolation network through in-situ-grown AgNPs that were reduced by DMF (supplementary test $\mathrm{S} 4)^{28,29}$. The presence of a reduction process in PANSion solutions was supported by a characteristic peak of ultraviolet-visible light (UV-Vis) spectra at 422 nm (Fig. 2b; and Supplementary Fig. 8) owing to the localized surface plasmon resonance effect of AgNPs ${ }^{30}$, of which the intensity was notably correlated with the heating time (Fig. 1j). Thus, in light of these properties, we sought to obtain PANSion film that was conductive, stretchable, and sufficiently strong to withstand mechanical disturbances when deployed onto the human body or robotics for sensing and actuation applications.

We applied a solution casting approach with thermal drying to prepare the freestanding PANSion film (Fig. 2a) that was partially visible-light-transparent (thickness-dependent, Supplementary Fig. 9). It should be emphasized that the container during the drying process was open-ended to avoid the formation of a gel (Supplementary Fig. 7c). Under thermal heating (e.g., $\left.55^{\circ} \mathrm{C}\right)$, the DMF evaporation rate was accelerated, and $\left[\operatorname{Ag}(-\mathrm{C} \equiv \mathrm{N})_{x}\right]^{+}$complexes were formed. Thus, the volume-normalized cross-linking density within the PANSion film was much higher than that within its gel counterpart, resulting in an extensively entangled network with high strength. Fig. 2c and d present the scanning electron microscopy (SEM) images of PAN and PANSion films which show morphologies of a grainy structure and smooth surface, respectively. Such structure contrast may stem from their crystalline differences where PAN is semicrystalline and PANSion is amorphous (Supplementary Fig. 10). Note that no AgNPs clusters at the microscale were found on the SEM images (namely, no aggregations). Additionally, the element distributions of linescan and mapping profiles based on energy-dispersive X-ray spectroscopy (EDS) further supported the notion of an evenly spread of silver (Fig. 2e; and Supplementary Fig. 11). For comparison, no trace silver was detected in the PAN film using either EDS or X-ray photoelectron spectroscopy (XPS), corroborating expected results (Supplementary Fig. 12-13).

Furthermore, the presence of in-situ-grown AgNPs was directly validated using transmission electron microscopy (TEM) images, where a notable scattering with an average lateral size of $\sim 10$ nm was observed (Fig. 2f; and Supplementary Fig. 14). Consequently, the as-prepared PANSion film, when used as a single homogeneous material unit, was expected to be electrically percolated 
by AgNPs, bringing an improved electrical conductivity to PANSion, despite the inherent insulating nature of PAN. We further substantiated this claim of the in-situ-created electron conductive pathway by carrying out nanoscale-level conductive atomic force microscopy (CAFM). As revealed in Fig. 2g, C-AFM results illustrated the transformation of the material from electrically insulating to percolatively functional current pathways when $\phi$ varies from 0 to 2.0. Especially, PANSion film of $\phi=2.0$ exhibited the most noticeable change in helping to establish conductive networks. With respect to conventional add-on approaches used to construct conductive, soft, and stretchable composites, for instance, mixing AgNPs with elastomers or coating AgNPs on elastomers' surface, our PANSion via a built-in approach was shown to be far superior to creating a silver-polymer framework. By relying on PANSion, we were able to ultimately devise a methodology that is ideal on the fronts of dispersion quality, structure control and fabrication simplicity. Furthermore, we accomplished a seamless integration of both improved conductivity and stretchability in a single homogeneous material unit in just one step: introducing $\left[\mathrm{Ag}(-\mathrm{C} \equiv \mathrm{N})_{x}\right]^{+}$complexes with concomitant in-situ-grown AgNPs. Taken together of the coupled properties, we substantiate that the high stretchability of PANSion at the molecule level originated from $\left[\operatorname{Ag}(-\mathrm{C} \equiv \mathrm{N})_{x}\right]^{+}$complexes, whilst the improved conductivity at nanoscale rested on the in $^{-}$ situ-grown AgNPs, collectively empowering PANSion film with sufficient strength (for actuation) and multimodal sensing capabilities (for sensing) (discussed in following sections).

\section{Mechanical properties of PANSion film}

Having studied PANSion material properties at the molecular, nano, and microscale, we continued our studies to probe on macroscale mechanical performance. Fig. 3a shows the stretchingreleasing of a PANSion film, suggesting a high stretchability and good strain recovery (Supplementary Fig. 15). Additionally, no cracks at the microscale were found on optical images taken during stretching (Supplementary Fig. 16). The highest stretchability (i.e., failure strain) exceeded $1000 \%$ for PANSion of $\phi=2.0$, which is a $>500 \times$ improvement of elasticity compared to the control PAN (Fig. 3b; and Supplementary Fig. 17a). Moreover, Young's modulus ( $>20$ $\mathrm{kPa}$ ) was sufficiently low and enabled conformal fitting for curved surfaces (Supplementary Fig. 17b). On one front, the formation of $\left[\mathrm{Ag}(-\mathrm{C} \equiv \mathrm{N})_{x}\right]^{+}$complexes produced a structural change of crystallinity, leading to reduction of both strength and modulus (Supplementary Fig. 17c), 
potentially due to the disorderliness of the viscoelastic PANSion network and its low glass transition temperature ( $\left.T_{\mathrm{g}}\right)$ (Supplementary Fig. 18). On another front, the residual solvent, DMF, also inexplicitly promoted PANSion chains' superelasticity as a lubricant (Supplementary Fig. 19 and Supplementary Table 4) ${ }^{31,32}$. As previously discussed, the energy dissipation owning to mechanical ruptures of $\left[\mathrm{Ag}(-\mathrm{C} \equiv \mathrm{N})_{x}\right]^{+}$complexes was again confirmed by the stress-strain hysteresis measured during continuous stretching-releasing cycles from $20 \%$ to $300 \%$ (Fig. 3c; and Supplementary Fig. 17d and e). Considering these findings, we estimate that stretching- and releasing-associated network extension-contraction cycles were brought about by the dissociation and reformation of $\left[\mathrm{Ag}(-\mathrm{C} \equiv \mathrm{N})_{x}\right]^{+}$complexes together with molecular chain straightening (Supplementary Fig. 20). To validate this hypothesis, we performed computational calculations via first principles to help rationalize the reasoning behind the observed gained stretchability (Supplementary Text 1).

Having said that, perceptions on the samples' structural evolutions were carried out using coarsegrained molecule dynamic (CG-MD) simulations, which provided insights on the PANSion chain conformation and dynamics of $\left[\mathrm{Ag}(-\mathrm{C} \equiv \mathrm{N})_{x}\right]^{+}$complexes during stretching. Unlike the semicrystalline PAN, the constructed model of PANSion, with an undeliberately regulated AgNPs distribution, showed an amorphous structure that agreed with our experimental observations (Fig. 3d, Supplementary Fig. 21). Fig. 3e presents the PANSion network's evolution when the applied stretch, $\varepsilon$, was escalated from 1 to 5 (i.e., 100 to 500\%). The network extension and sliding can justify the superelastic properties of the PANSion film (Supplementary Video 1). Concomitantly, chain alignment was also monitored (Fig. 3f), where a larger strain produced a higher alignment degree, corresponding to a large value of Herman's orientation factor $\left(f_{(\mathrm{H})}\right)^{25}$. For instance, an $f_{(\mathrm{H})}$ value of 0.3427 for $\varepsilon=1$, while an $f_{(\mathrm{H})}$ value 0.6331 for $\varepsilon=5$, seems to substantiate our initial hypothesis ${ }^{23}$. The non-overlapping of $f_{(\mathrm{H})}$ between the first and second stretching-releasing cycles might also have arisen from the strain hysteresis or reformation of $\left[\mathrm{Ag}(-\mathrm{C} \equiv \mathrm{N})_{x}\right]^{+}$complexes at different sites. Intriguingly, no mechanical mismatch of compliance between AgNPs and their surrounding matrix was noted during stretching (Fig. 3g), a welcomed finding considering the desired mechanical durability needed for PANSion-based electronics. It must be pointed out that post-added inorganic fillers usually deteriorate the softness or stretchability of the polymer matrix 
due to strengthening effects. In contrast, PANSion with in-situ-grown AgNPs still exhibited low modulus and sufficient strength with high elasticity.

On a final note, we mapped the density distribution of $\left[\mathrm{Ag}(-\mathrm{C} \equiv \mathrm{N})_{x}\right]^{+}$complexes at different strains, which seemingly does not seem to change (Fig. 3h, Extended Data Fig. 4). Such a phenomenon can be attributed to the dynamics of $\left[\mathrm{Ag}(-\mathrm{C} \equiv \mathrm{N})_{x}\right]^{+}$complexes during stretching, where dissociation and reformation prevail simultaneously. Over $90 \%$ of complex bonds were newly regenerated as strain reached beyond $200 \%$ and was even higher for further stretching cycles (Fig. 3i). In brief, the obtained stretchability of PANSion can be ascribed to the introduction of $\left[\mathrm{Ag}(-\mathrm{C} \equiv \mathrm{N})_{x}\right]^{+}$complexes that bring disorderliness and dynamic network entanglement alongside dissociation-reformation events. Together with percolation conductivity from in-situ-grown AgNPs, these two synchronous features encourage PANSion's use in soft and stretchable electronics, as well as in soft robotics as functional artificial tissues.

\section{Multimodal sensing of PANSion for soft electronics}

As a proof-of-concept, we first explored the piezoresistive sensing using PANSion film (Supplementary Text 5), taking advantage of its softness ( $\sim 220 \mathrm{kPa}$ in modulus), stretchability ( 1200\% strain), and conductivity $\left(0.076 \mathrm{~S} \mathrm{~m}^{-1}\right)$ (Supplementary Figs. 22-25). The PANSion sensor was implemented to monitor various body movements such as elbow bending, foot walking, and finger wagging with an acceptable sensitivity, good stability, and fast response (85 ms for responding and $0.5 \mathrm{~s}$ for recovering) (Fig. 4a-b, Supplementary Figs. 26-28). Taking a further step, PANSion solutions (i.e., $10 \mathrm{wt} \%$ of PAN, $\phi=2.0$ ) can be utilized for dip-coating on flexible substrates (i.e., fabrics), yielding sensing textiles that can be used for motion detections (Supplementary Fig. 29-32, while still maintaining their original softness and stretchability (Fig. 4c top, Extended Data Fig. 5). Intriguingly, this versatile application was further endorsed by the direct spinning of PANSion fibers in the air, attainable when its viscosity was appropriately tuned (Fig. 4c bottom). As one subject of our future studies, PANSion fiber, inheriting stretchability, conductivity, and sensitivity from its film antetype (Extended Data Fig. 6), could provide a platform technology to be used as a basic building block for intelligent textiles ${ }^{33,34}$. 
Furthermore, PANSion's multistimuli response properties were examined, showing two sensing modes of capacitive and piezoresistive mechanisms (Supplementary Text 6; and Supplementary Fig. 33-34). Fig. 4d illustrates the device structure with two electrodes and its corresponding sensing mechanisms when subjected to physical touch, thermal exposure, and mechanical disturbances that represent three primary natural stimuli that biological tissues encounter every day. After conformally wrapping a prosthetic finger with a PANSion film as e-skin (Fig. 4e), capacitance-modal sensing could be exploited to identify the presence of a pulse or static touch (Fig. 4f; and Supplementary Video 2), and even differentiate between different touch sources, depending on signal features (i.e., metal bar and human finger, Fig. $4 \mathbf{f}$ inset). Such a platform could bestow robotics components skin-like intelligent proprio- and exteroceptive capabilities, especially when coupled alongside specific machine learning algorithms used for signal analysis (Supplementary Fig. 35). Resistance-modal sensing is suitable for detecting thermal disturbances. Thus, PANSion e-skin can be used to allow a prosthetic finger to "feel" nearby cold or warm objects (Supplementary Fig. 36-37), including human respiration, as associated to bodytemperature exhaled breath detection (Fig. $4 \mathbf{g}-\mathbf{h}$; and Supplementary Fig. 38, Supplementary Video 2). Note that one limitation for the current design is the detection of objects with a thermal profile similar or close to that of PANSion e-skin (i.e., the environment temperature) when approached or even contacted ${ }^{35}$.

Additionally, we demonstrated PANSion e-skin's ability for finger touch behavior differentiation, including simple touch (gentle contact in the form of a thermal stimulus), as well as touch-andstretch (full contact as thermal and strain stimuli) (Fig. 4i). A resistance drop occurred when the human finger (thermal source, $\sim 36.5^{\circ} \mathrm{C}$ ) came into proximity with the PANSion e-skin. This is shown as the "touch" stage in Fig. 4j. A plateau of resistance change took place when performing the "touch and hold" stage because the thermal exchange reached equilibrium (Supplementary Fig. 39). If any strain stimuli (i.e., stretch or shear) are practiced during touch, PANSion e-skin can also perceive that mechanical disturbance as portrayed by distinct electrical signals, including small and large stretches (Fig. 4j inset). Such multifunctionality of PANSion e-skin based on multimodal sensing mechanisms enables versatile applications for soft and stretchable electronics onto the human body, as well as in robotic systems. 


\section{Self-sensing actuator using PANSion membrane}

In addition to the aforementioned applications, we also explored PANSion's potential as a sensorized pneumatic actuator for soft robotics applications (Supplementary Text 7). It is worth highlighting that our PANSion actuator, benefiting from in-situ-grown AgNPs, is self-sensing, a feature usually absent for previously reported cases, or which requires additional post-treatments to be achieved (e.g., surface conductive coating) ${ }^{5,12,19}$. Fig. 5a illustrates the actuator design where a PANSion membrane was connected to a cone-shaped pneumatic circuit at the perimeter. Pressure within the chamber was increased $(\Delta P>0)$ to activate the actuator, resulting in PANSion membrane deformation with a displacement $(\Delta d)$ and output force $(F)$ (Fig. 5b). Here the applied strain (i.e., areal strain) on the PANSion actuator due to pressure change was two-dimensional (2D) instead of 1D. The actuation performance was analyzed by plotting displacement, output force, and areal strain versus pressure (Fig. 5c, and Extended Data Fig. 7). In the current design, the displacement could reach $\sim 20 \mathrm{~mm}(\sim 184 \%$ areal strain $)$ for outward direction $(\Delta P>0)$ with a maximum output force at $\sim 3.6 \mathrm{~N}$. Similar actuation performance was observed for inward direction $(\Delta P<0)$. When being actuated, the displacement characteristics, including amplitude (large/small) and frequency (high/low), were readily detected by monitoring the PANSion's piezoresistive behavior (Fig. 5d and e; Supplementary Fig. 40 and Supplementary Video 3). These electrical signals could be further collected as inputs to enable a more dedicated control on PANSion actuators for specific tasks in future studies.

Concept validation of using a self-sensing PANSion actuator as an artificial muscle was also demonstrated for weight-lifting tasks, where a negative pressure $(\Delta P<0)$ was applied, resulting in an inward displacement (Fig. 5g). Fig. 5h presents snapshots of a sample used to lift a 50-g load to a height of $\sim 15 \mathrm{~mm}$ (Supplementary Video 4). Remarkably, this setup could also lift a 100-g load cyclically (Supplementary Video 5), a load at least 3700x its own weight. With this pneumatic design, the PANSion actuator exhibited a maximum specific work of at least $350 \mathrm{~J} \cdot \mathrm{kg}^{-}$ ${ }^{1}$ (Fig. 5j) and power density of $\sim 467 \mathrm{~W} \cdot \mathrm{kg}^{-1}$ that were comparable with those of other state-ofart soft actuators based on synthetic materials, even surpassing natural tissues (Extended Data Fig. 1, and Supplementary Table 1). By controlling pressure, natural actuation patterns from muscles could be reproduced on our PANSion actuators, such as lifting-releasing, lifting-holding, and lifting-controlled releasing (Supplementary Video 5). What may attract more attention are 
351 the distinct electrical signals obtained when performing different actuation behaviors (Fig. 5i, and

352 Supplementary Fig. 41) or manipulating objects at different temperatures (Fig. 5k-m; and

353 Extended Data Fig. 8 and Supplementary Video 6). These could indeed be harnessed, as

354 preliminary inputs of proprioception and exteroception signals, to monitor and control robotics'

355 activity deliberately. For instance, Fig. 5f exhibited recognizable signals for actuation with and

356 without loads that could be explored to endow robotics with sensing capabilities similar to that of

357 biological tissues incurred when performing weight-lifting of large ("heavy"), small ("light"), and

358 zero ("feather") loads. Such seamless unification of sensing and actuation in a single homogeneous

359 material system is a necessary step toward achieving closed-loop feedback control of soft robots,

360 machines, and haptic devices.

\section{Outlook}

362 In addition to in-situ-grown $\operatorname{AgNPs}$ and $\left[\operatorname{Ag}(-\mathrm{C} \equiv \mathrm{N})_{x}\right]^{+}$coordination complexes, we report a silver-

363 polymer-framework-based elastomer that is conductive, soft, stretchable, yet sufficiently strong.

364 Dual functions in a single homogeneous material platform were realized on PANSion: multimodal

365 sensing based on capacitive and piezoresistive mechanisms (for e-skin and other body-integrated

366 electronics with conformal fitting) and sensorized soft actuator (for intelligent robotics with life-

367 like sensing capabilities). PANSion technology represents one of the many steps for artificial

368 materials with seamless integration of sensation and actuation capability on the path towards

369 reproducing full sensorimotor-like functions natively found in biological tissues. The material we

370 present can respond only to mechanical and thermal stimuli while actuating; what remains an

371 opportunity is the inclusion of other sensing capabilities such as perspiration, gustation, etc. 


\section{References}

373 1. Wang, M. et al. Artificial skin perception. Adv. Mater. 33, 2003014 (2020).

374 2. Zhao, H., O'Brien, K., Li, S. \& Shepherd, R. F. Optoelectronically innervated soft

3. Pena-Francesch, A., Jung, H., Demirel, M. C. \& Sitti, M. Biosynthetic self-healing

4. Baumgartner, M. et al. Resilient yet entirely degradable gelatin-based biogels for soft robots and electronics. Nat. Mater. 19, 1102-1109 (2020).

5. Kanik, M. et al. Strain-programmable fiber-based artificial muscle. Science 365, 145-150 (2019).

6. Soon, R. H., Yu, L. \& Lim, C. T. A soft sensorized microfluidic tubular actuating gripper. Adv. Mater. Technol. 5, 2000150 (2020).

7. Acome, E. et al. Hydraulically amplified self-healing electrostatic actuators with musclelike performance. Science 359, 61-65 (2018).

8. $\mathrm{Wu}, \mathrm{X}$. et al. A potentiometric mechanotransduction mechanism for novel electronic skins. Sci. Adv. 6, eaba1062 (2020).

9. Cao, Y. et al. Self-healing electronic skins for aquatic environments. Nat. Electron. 2, 75-82 (2019).

10. Su, Y. et al. Muscle fibers inspired high-performance piezoelectric textiles for wearable physiological monitoring. Adv. Funct. Mater. 31, 2010962 (2021).

11. Dai, Y., Hu, H., Wang, M., Xu, J. \& Wang, S. Stretchable transistors and functional circuits for human-integrated electronics. Nat. Electron. 4, 17-29 (2021).

12. Wang, J., Gao, D. \& Lee, P. S. Recent progress in artificial muscles for interactive soft robotics. Adv. Mater. 33, 2003088 (2021).

13. Rich, S. I., Wood, R. J. \& Majidi, C. Untethered soft robotics. Nat. Electron. 1, 102-112 (2018).

14. Choi, S. et al. Highly conductive, stretchable and biocompatible ag-au core-sheath nanowire composite for wearable and implantable bioelectronics. Nat. Nanotechnol. 13, 1048-1056 (2018).

15. Chun, K. Y. et al. Highly conductive, printable and stretchable composite films of carbon nanotubes and silver. Nat. Nanotechnol. 5, 853-857 (2010).

16. Liu, Z. et al. Highly stable and stretchable conductive films through thermal-radiationassisted metal encapsulation. Adv. Mater. 31, 1901360 (2019).

17. Ma, R., Kang, B., Cho, S., Choi, M. \& Baik, S. Extraordinarily high conductivity of stretchable fibers of polyurethane and silver nanoflowers. ACS Nano 9, 10876-10886 (2015).

18. Boland, C. S. et al. Sensitive electromechanical sensors using viscoelastic graphenepolymer nanocomposites. Science 354, 1257-1260 (2016).

19. Zhao, Y. et al. Somatosensory actuator based on stretchable conductive photothermally responsive hydrogel. Sci. Robot. 6, eabd5483 (2021).

20. Truby, R. L. et al. Soft somatosensitive actuators via embedded $3 d$ printing. Adv. Mater. 30, 1706383 (2018).

21. Chen, L. et al. Graphene-based actuator with integrated-sensing function. Adv. Funct. Mater. 29, (2019). 
22. Ford, M. J. et al. A multifunctional shape-morphing elastomer with liquid metal inclusions. Proc. Natl. Acad. Sci. 116, 21438-21444 (2019).

23. Zhang, S., Hao, A., Liu, Z., Park, J. G. \& Liang, R. A highly stretchable polyacrylonitrile elastomer with nanoreservoirs of lubricant using cyano-silver complexes. Nano Lett. 19, 3871-3877 (2019).

24. Li, C. H. et al. A highly stretchable autonomous self-healing elastomer. Nat. Chem. 8, 618-624 (2016).

25. Zhao, X. et al. Bioinspired ultra-stretchable and anti-freezing conductive hydrogel fibers with ordered and reversible polymer chain alignment. Nat. Commun. 9, 3579 (2018).

26. $\mathrm{Li}, \mathrm{C}$. et al. Supramolecular-covalent hybrid polymers for light-activated mechanical actuation. Nat. Mater. 19, 900-909 (2020).

27. Wu, Y. et al. Bioinspired supramolecular fibers drawn from a multiphase self-assembled hydrogel. Proc. Natl. Acad. Sci. 114, 8163-8168 (2017).

28. Pastoriza-Santos, I. \& Liz-Marzán, L. M. Formation and stabilization of silver nanoparticles through reduction by n,n-dimethylformamide. Langmuir 15, 948-951 (1999).

29. Pastoriza-Santos, I. \& Liz-Marzán, L. M. N,n-dimethylformamide as a reaction medium for metal nanoparticle synthesis. Adv. Funct. Mater. 19, 679-688 (2009).

30. Parente, M. et al. Simple and fast high-yield synthesis of silver nanowires. Nano Lett. 20, 5759-5764 (2020).

31. Campanella, A., Dohler, D. \& Binder, W. H. Self-healing in supramolecular polymers. Macromol. Rapid. Commun. 39, 1700739 (2018).

32. Khare, E., Holten-Andersen, N. \& Buehler, M. J. Transition-metal coordinate bonds for bioinspired macromolecules with tunable mechanical properties. Nat. Rev. Mater. 6, 421436 (2021).

33. Zhang, S. et al. Leveraging triboelectric nanogenerators for bioengineering. Matter 4, 845-887 (2021).

34. Zhou, Z. et al. Sign-to-speech translation using machine-learning-assisted stretchable sensor arrays. Nat. Electron. 3, 571-578 (2020).

35. You, I. et al. Artificial multimodal receptors based on ion relaxation dynamics. Science 370, 961-965 (2020). 


\section{Methods}

Materials. Polyacrylonitrile (PAN, average $M w$ 150,000), $N, N$-Dimethylformamide (DMF, $99.8 \%)$, silver nitrate $\left(\mathrm{AgNO}_{3}, \mathrm{ACS}\right.$ reagent, $\left.\geqslant 99.0 \%\right)$, calcium chloride $\left(\mathrm{CaCl}_{2}\right.$, anhydrous, powder, $\geqslant 97 \%)$, magnesium sulfate heptahydrate $\left(\mathrm{MgSO}_{4} \cdot 7 \mathrm{H}_{2} \mathrm{O}, \mathrm{ACS}\right.$ reagent, $\left.\geqslant 98.0 \%\right)$, zinc chloride (ACS reagent, $\geqslant 97.0 \%)$, iron (II) chloride tetrahydrate $\left(\mathrm{FeCl}_{2} \cdot 4 \mathrm{H}_{2} \mathrm{O}\right.$, reagent grade, $\geqslant$ $98.0 \%)$, copper(II) sulfate pentahydrate $\left(\mathrm{CuSO}_{4} \cdot 5 \mathrm{H}_{2} \mathrm{O}\right.$, ACS reagent, $\left.\geqslant 98.0 \%\right)$, were purchased from Sigma-Aldrich. All chemicals were used as received without any post-treatment.

\section{Preparations of PANSion solutions and free-standing film. DMF was used as a solvent to obtain} a well-dissolved PAN solution first (Hotplate/Stirrer, VWR, $500 \mathrm{rpm}$ for $24 \mathrm{~h}$ ), of which the base concentration was fixed at $2 \mathrm{wt} \%$. All PANSion solutions were prepared by adding varying amounts of $\mathrm{AgNO}_{3}$ into the PAN solutions mentioned above (stirring for $0.5 \mathrm{~h}$ ). In this study, the weight ratio of $\mathrm{AgNO}_{3}: \mathrm{PAN}, \phi$, was adjusted from 0.5 to 2.0 (Supplementary Text 1, and Supplementary Table 3). The control sample included the $2 \mathrm{wt} \%$ PAN solution $(\phi=0)$. PANSion solutions were contained in sealed vials for observing the PANSion gelation transition. To obtain free-standing PANSion film, a specific volume of PANSion solutions (e.g., $10 \mathrm{~mL}$ ) was transferred into a PTFE mold for thermal drying (oven heating at $55^{\circ} \mathrm{C}$ ), where the temperature was intentionally controlled.

Conductive atomic force microscopy (C-AFM). Thin PANSion films prepared by drop-casting $(200 \mu \mathrm{L})$ on silicon wafers with subsequent oven drying (at $55^{\circ} \mathrm{C}$ ) were used for C-AFM measurements. All tests were performed on a Bruker Dimension Icon Scanning Probe Microscopy (Bruker Nano Surfaces Division, Singapore), equipped with a cantilever of a resonance frequency of $\sim 20 \mathrm{kHz}$, spring constant at $0.4 \mathrm{~N} \mathrm{~m}^{-1}$, and probe tip radius at $\sim 20-50 \mathrm{~nm}$. During C-AFM scanning, both topography and current mapping results could be obtained. For PAN $(\phi=0)$ and PANSion of $\phi=0.5,1.0$, and 1.5, a fixed bias of $150 \mathrm{mV}$ was applied. Note that an external resistor was loaded as a current divider for $\phi=2.0$ (since it was relatively more conductive than other samples) to avoid heat accumulation at the probe. All measurements were conducted at room temperature of $\sim 23^{\circ} \mathrm{C}$.

AFM-based single-molecule force spectroscopy (AFM-SMFS). Atomic force microscopybased single-molecule force spectroscopy (AFM-SMFS) experiments were performed on 
Nanowizard 4 AFM (JPK Germany) at room temperature $\left(\sim 25^{\circ} \mathrm{C}\right)$. AFM cantilever (MLCT-BioDC, Bruker, United States) with a spring constant ( $k$ ) of $\sim 40 \mathrm{pN} \mathrm{nm}^{-1}$ was used. The equipartition theorem was used to calibrate the $k$ of each cantilever in solution with an accurate value before the experiment. Both PAN and PANSion solutions in sealed vials were subjected to heating at $55{ }^{\circ} \mathrm{C}$ for 48 hours before the test. An evaporation blocker was used to prevent solvent evaporation, thus maintaining a stable test condition by minimizing the air disturbance. Typically, an $800 \mu \mathrm{L}$ solution was added on a clean glass coverslip for AFM-SMFS measurement. First, the cantilever contacted the surface with an indentation force of $\sim 1 \mathrm{nN}$ for $1 \mathrm{~s}$ and randomly picked up a molecule. Then, the cantilever moved up vertically in which the captured PNA or PANSion molecule was stretched. Finally, the molecule broke upon further stretching leading to a rupture force peak in the forceextension curve. The data analysis was carried out using the program Igor Pro 6.12 (WaveMetrics Inc.). The curves were fitted with the worm-like-chain (WLC) model of polymer elasticity.

Coarse-grained molecular dynamic (CG-MD) simulation. As illustrated in Supplementary Fig. 24a, in the CG model of PAN, each bead, named type A, represents a monomeric unit of PAN. An $\mathrm{Ag}^{+}$ion was modeled along with a $\mathrm{NO}_{3}{ }^{-}$as one bead, assigned as type $\mathrm{B}$. The cluster of $\mathrm{Ag}$ was modeled as a spherical rigid body of $3 \mathrm{~nm}$ that was uniformly packed by $60 \mathrm{C}$ beads at the surface. The residual solvents are implicated in our model system. The force field of the CG PAN model was developed based on that of the CG PVA (polyvinyl alcohol) model, which was built up previously ${ }^{36-38}$. Specifically, the potential functions and the parameters of intramolecular interactions in the PAN model are set the same as that of PVA since the two polymers share the same backbone. The parameters of inter-molecular non-bonded interactions are refitted according to the binding energies calculated by density functional theory between PAN and PAN and between PAN and $\mathrm{Ag}^{+}$ion. With the force field, the simulated crystallinity of pure PAN (48\%) smaller than that of PVA $(56 \%)$ was reasonable ${ }^{39}$. The degree of crystallization was evaluated by the ratio of beads with the value of $\mathrm{Q}_{6}$ exceeding $0.2^{40}$. The $\mathrm{CG}$ simulations use a set of reduced units where the length unit was $\sigma_{0}=0.52 \mathrm{~nm}$, corresponding roughly to the diameter of PAN repeat unit, the energy unit was $\epsilon_{0}=\mathrm{k}_{\mathrm{B}} \mathrm{T}$ with $\mathrm{T}=550 \mathrm{~K}$, and the mass unit was the mass of PAN repeat unit, i.e., $m=53 \mathrm{amu}$. The time unit was thereby estimated as $\tau_{0}=1.63 \mathrm{ps}$. The non-bonded interactions between CG beads are given by Lennard-Jones (9-6) potential 


$$
V_{\mathrm{LJ}}(r)=6.75 \epsilon_{i j}\left[\left(\frac{\sigma_{i j}}{r_{i j}}\right)^{9}-\left(\frac{\sigma_{i j}}{r_{i j}}\right)^{6}\right],
$$

where $r_{i j}$ was the distance between bead $i$ and bead $j$, the interaction parameters $\epsilon_{i j}$ and $\sigma_{i j}$ are listed in Supplementary Table 5. This potential was cut off at $r_{\text {cut }}=3.0 \sigma_{0}$ under NPT ensemble (at 1 atm). The harmonic potential was employed to describe bond stretching interactions,

$$
V_{\text {bond }}(r)=\frac{1}{2} K_{\text {bond }}\left(r-r_{0}\right)^{2} \text {, }
$$

where $r_{0}=0.5 \sigma_{0}$ and $K_{\text {bond }}=2704 \epsilon_{0} / \sigma_{0}^{2}$. The angle bending interactions for PAN molecules are described by a numerical potential given by ref. ${ }^{37}$, which was obtained by bottom-up coarsegraining from all-atom simulations.

Electrical conductivity and sensing performance characterization. Electrical conductivity was assessed using the two-wire method using Keithley Model 2450. The resistance change $(\Delta R / R(\%))$ vs. strain was obtained by continuously recording the resistance data while stretching the PANSion film using a linear motor. The stability of resistance change at $200 \%$ strain was assessed by holding the strain for $\sim 30 \mathrm{~min}$. The piezoresistive sensing performance of PANSion film was assessed using a linear motor to apply specific strains (from $1 \%$ to $400 \%$ strain) while recording resistance data. At each testing strain, at least 10 cycles were repeated. PANSion film was attached to the corresponding body region such as finger joint, elbow, and ankle joint for motion detections. PANSion coated fabric was sewed into a sleeve around the elbow area so that it could easily monitor the elbow bending motion. PANSion fiber was demonstrated to be strain sensitive by hand-stretching while fixing the fiber's two ends. A prosthetic finger wrapped with PANSion film as an artificial "skin" to detect different stimuli was demonstrated where capacitance mechanism $(\triangle C / C(\%))$ was applied for touch detection, including metal bar and a human finger. Cold $(\sim 2-5$ $\left.{ }^{\circ} \mathrm{C}\right)$ and hot water $\left(\sim 70{ }^{\circ} \mathrm{C}\right)$ in a vial were used as thermal stimuli to validate the temperature detection capability. Expiration was directed to the prosthetic finger for breathing out detection.

Self-sensing actuation characterization. A pneumatic-mechanism-based actuator was built for the application demo of PANSion since this type of actuator was well investigated and has broad applications such as object grasping, transferring, and weight-lifting. A PANSion membrane was connected to the periphery of the cone-shape chamber $(\varnothing=50 \mathrm{~mm})$, constituting a single-chamber actuator. The pressure, indicated by a DP-101-E-P Panasonic pressure sensor, was adjusted controllably, leading to the PANSion membrane displacement (extracted manually from the video 
534 footage) with an output force (detected by a load cell from Trancell Technology inc., model BAB$53510 \mathrm{M})$. Thus, the relationship between displacement, output force, and areal strain of the PANSion 536 membrane with pressure was derived. A dead load of 50 or $100 \mathrm{~g}$ was glued to the PANSion 537 membrane via sticky tape (the contact area between tape and membrane was small, at $\sim 20 \mathrm{~mm}^{2}$ ).

538 An electrical signal $(\Delta R / R)$ was recorded simultaneously while performing the weight-lifting task 539 where specific energy and areal strain were calculated based on the lifting height (i.e., 540 displacement, $\Delta d$ ). For object manipulation (i.e., transferring) application, a soft and self-sensing 541 gripper was designed using a two-chamber configuration (cylinder-shaped, Supplementary Fig.

49a).

\section{Data availability}

All data are contained within the manuscript. Raw data are available from the corresponding authors upon reasonable request.

\section{References}

36. Luo, C. \& Sommer, J.-U. Coexistence of melting and growth during heating of a semicrystalline polymer. Physical Review Letters 102, 147801 (2009).

37. Reith, D., Meyer, H. \& Müller-Plathe, F. Mapping atomistic to coarse-grained polymer models using automatic simplex optimization to fit structural properties. Macromolecules 34, 2335-2345 (2001).

38. Sommer, J.-U. \& Luo, C. Molecular dynamics simulations of semicrystalline polymers: Crystallization, melting, and reorganization. Journal of Polymer Science Part B: Polymer Physics 48, 2222-2232 (2010).

39. Niu, W. et al. Remalleable, healable, and highly sustainable supramolecular polymeric materials combining superhigh strength and ultrahigh toughness. ACS Applied Materials \& Interfaces 12, 30805-30814 (2020).

40. Li, Y.-W. \& Sun, Z.-Y. The relationship between local density and bond-orientational order during crystallization of the gaussian core model. Soft Matter 12, 2009-2016 (2016).

\section{Acknowledgments}

The authors thank Fangfang Zhao at Bruker Nano Surface Division for C-AFM characterization and Yinnan Zhang at Singapore Management University for assistance on data visualization and 
563 representation. The work was supported by MOE Tier 2 R-284-000-217-112. P. Z. acknowledged 564 the National Natural Science Foundation of China (Grant No. 21771103, 21977047), Natural 565 Science Foundation of Jiangsu Province (No. BK20200058, BK20202004), the Fundamental 566 Research Funds for the Central Universities (Grant No. 14380205). J.C. acknowledged the Henry 567 Samueli School of Engineering \& Applied Science and the Department of Bioengineering at the 568 University of California, Los Angeles for the startup support.

\section{Author contributions}

570 S.Z., S.C.T., and J.C., conceived the idea for PANSion. S.Z., Y.-L.Z., S.C.T., and J.C. designed 571 the research. S.Z. developed the materials and methods for PANSion. Y.D. and P.Z. conducted the 572 SMFS experiment and analysis. S.Z. characterized the morphology property of AgNPs. W.S. 573 designed and conducted C-AFM characterization. J.Y. and L.Y. conducted XRD, DSC, and TGA 574 analysis. Y.-L.Z. conducted DFT calculation and MD simulation. S.Z. designed the rheological 575 measurements and conducted UV-vis spectrum absorbance analysis. All authors wrote the 576 manuscript.

577 Competing interests

578 A patent invented by S.Z. and S.C.T. is under application.

\section{Additional information}

580 Supplementary information is available for this paper. 

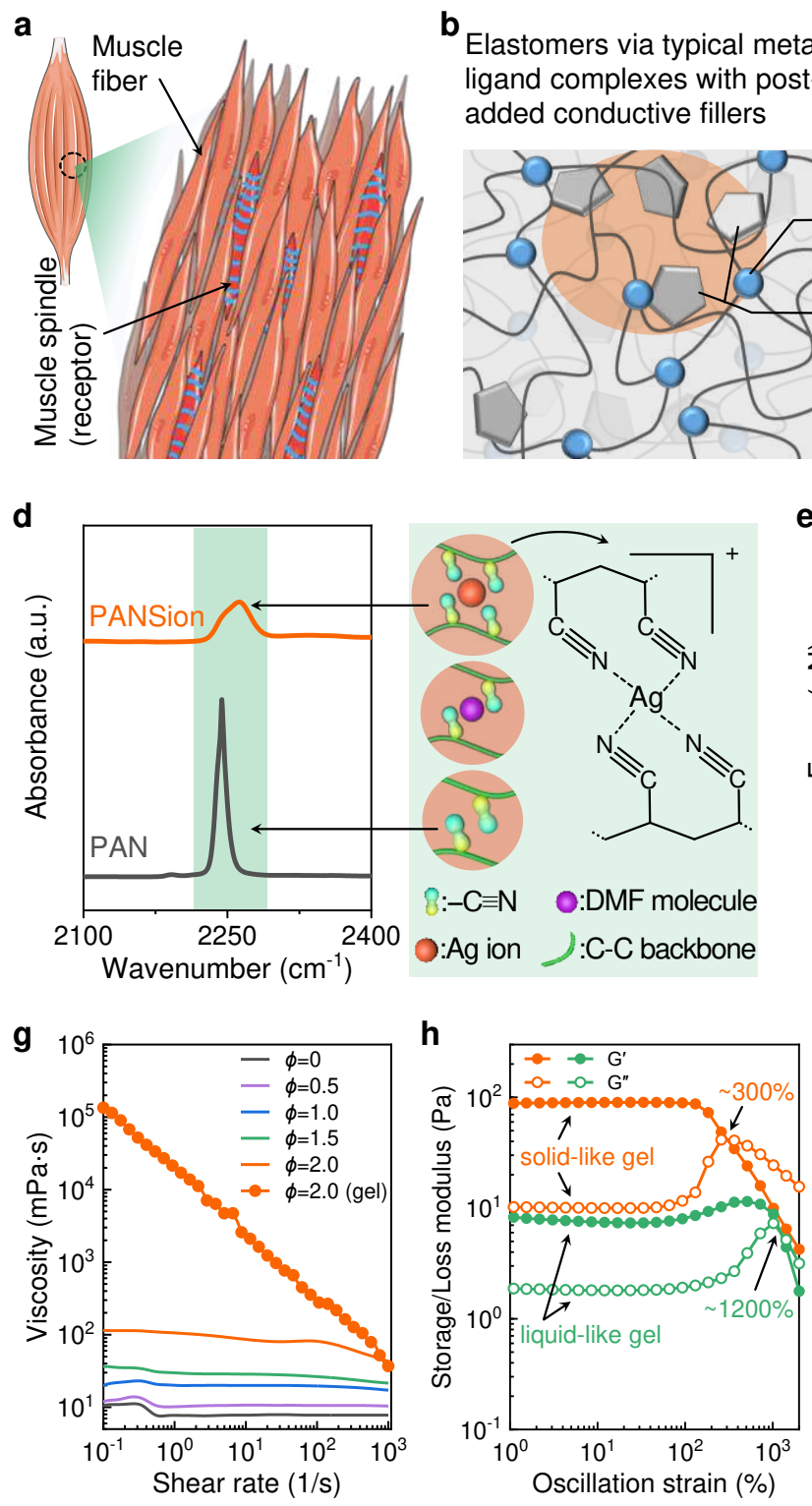

b Elastomers via typical metalligand complexes with postadded conductive fillers

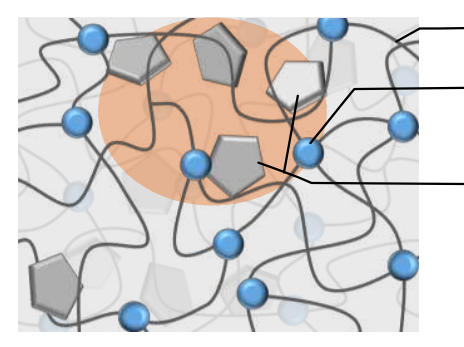
CANSion via silver-nitrile
complexes with
in-situ-grown AgNPs

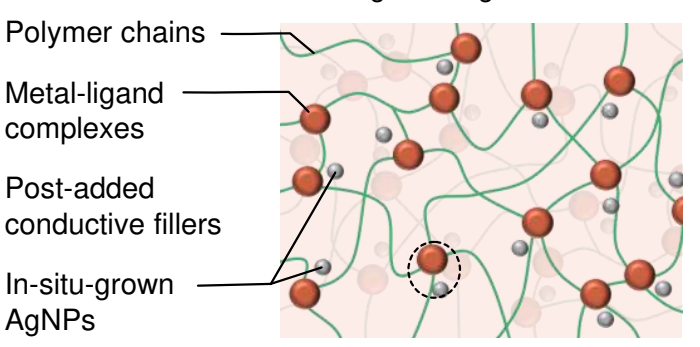

e
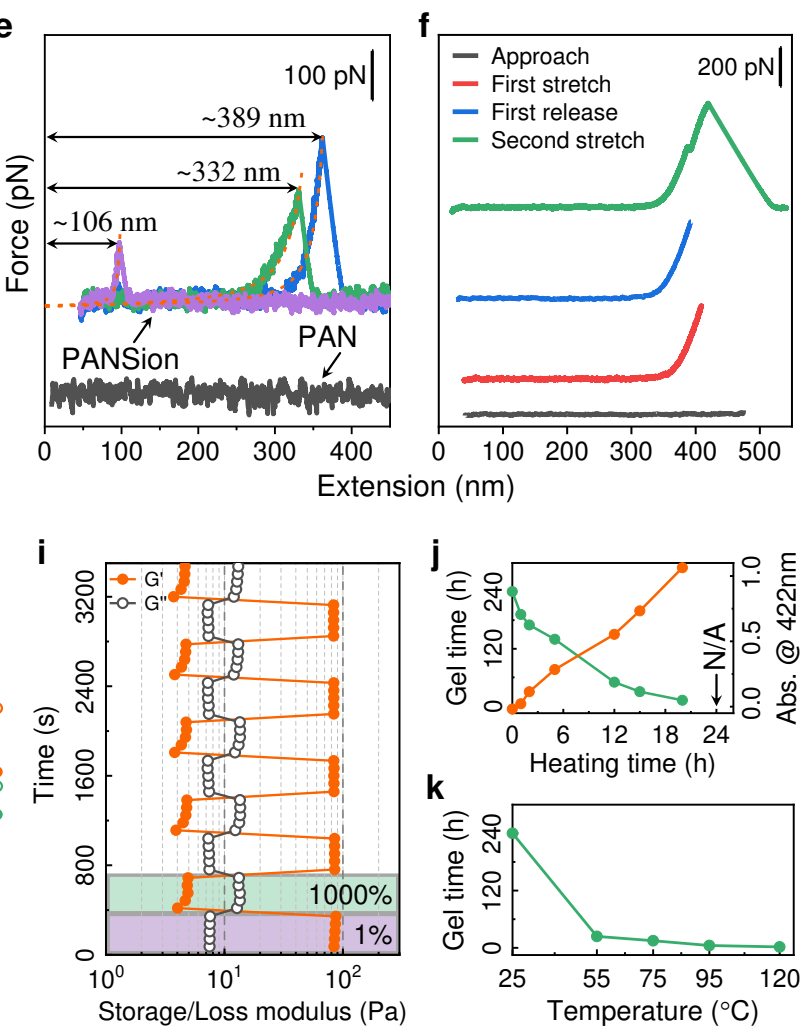

Fig. 1 Schematic of the structure design and mechanical properties of $\left[\operatorname{Ag}(-\mathrm{C} \equiv \mathrm{N})_{x}\right]^{+}$ complexes. (a) A schematic of natural tissue with muscle spindle (receptor) well distributed within the fiber-reinforced muscle. (b and $\mathbf{c}$ ) Schematics of conductive elastomers based on (b) a typical metal-ligand complex with post-added fillers and (c) the proposed silver-nitrile coordination complex with in-situ-grown AgNPs. (d) FTIR spectra (left) of PAN and PANSion showing different chemical environments of nitrile groups and schematics (right) for primary molecular interactions within the PANSion network. (e and $\mathbf{f}$ ) Representative force-extension curves of PAN and PANSion chains at the single-molecule level (e) and the stretchability of the PANSion chain facilitated by $\left[\operatorname{Ag}(-\mathrm{C} \equiv \mathrm{N})_{x}\right]^{+}$complexes (f). (g) Apparent viscosity of PANSion solutions with various $\phi$ values. Gelation significantly increased the viscosity $(\phi=2.0)$. (h) Oscillation-straindependent (1-2000\%) modulus changes of PANSion gel $\left(\phi=2.0, \omega=0.628 \mathrm{rad} \cdot \mathrm{s}^{-1}, 2{ }^{\circ} \mathrm{C}\right)$, 
594 showing a broad viscoelastic regime with a yielding strain beyond $100 \%$ (cross-over point at $595 \sim 300 \%$, yellow scatter-line curves). Green scatter-line curves present the mechanical responses of 596 liquid-like gel (cross-over point at $\sim 1200 \%$ ). (i) Step-strain oscillatory rheology of PANSion gel $597\left(\phi=2.0, \omega=0.628 \mathrm{rad} \cdot \mathrm{s}^{-1}, 25{ }^{\circ} \mathrm{C}, 5 \mathrm{cycles}\right)$. At $1 \%$ strain, storage modulus $\left(G^{\prime}\right)$ is leading over 598 loss modulus $\left(G^{\prime \prime}\right)$. After increasing to $1000 \%$ strain, both $G^{\prime}$ and $G^{\prime \prime}$ respond rapidly and can 599 nearly alternate to their initial values when strain is back to $1 \%$, demonstrating good recyclability. 600 (j and $\mathbf{k})$ Gel time of PANSion solutions $(\phi=2.0)$ as a function of heating time $(\mathrm{j})$ and temperature $601 \quad(\mathrm{k})$. 


\section{a}

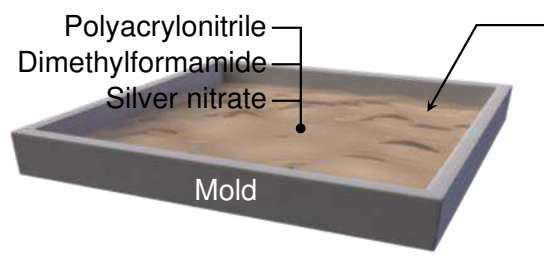

b

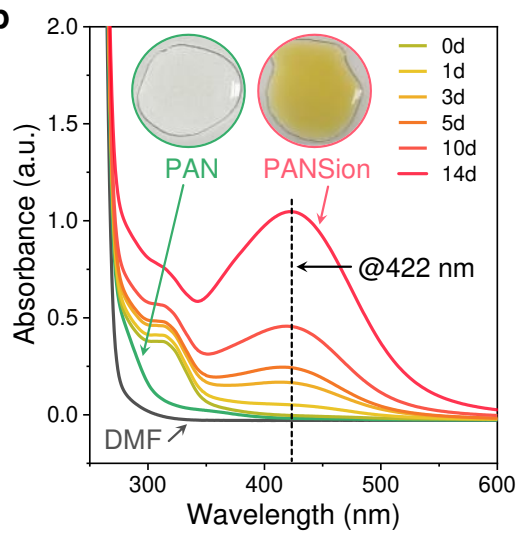

f

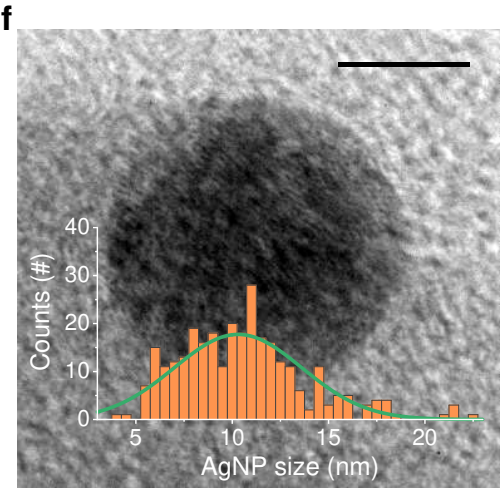

C

d
Casting solution

DMF evaporation at $55^{\circ} \mathrm{C}$

Metal-ligand complexes \& in situ AgNPs formation
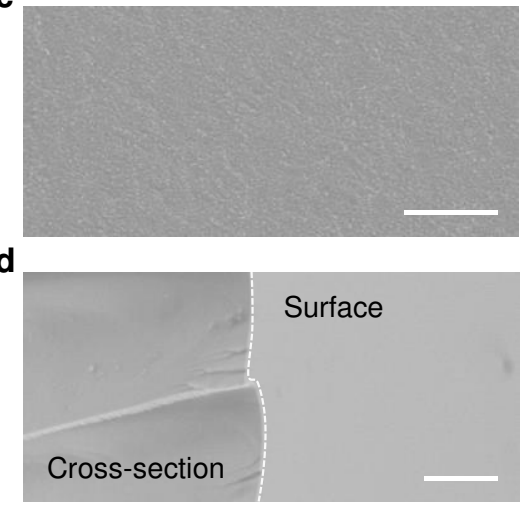

e

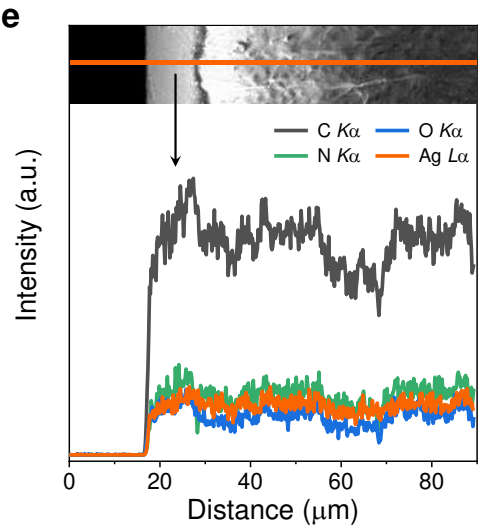

602

603

604

605

606

607

608

609

610

611

612

613 g
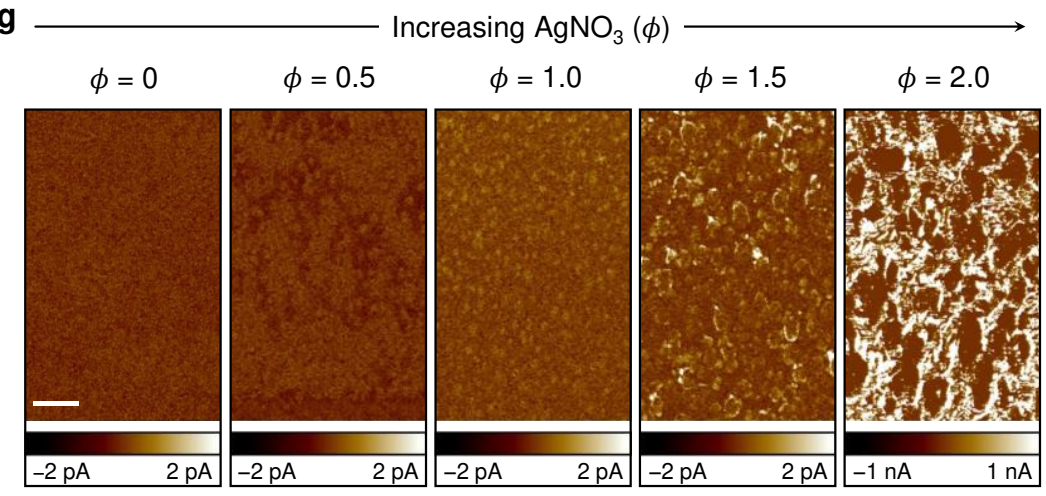

Fig. 2 Conductive PANSion from in-situ-grown AgNPs. (a) Schematic of PANSion film preparation. (b) UV-vis spectra of PANSion solutions $(\phi=2.0)$ with an absorbance peak at 422 $\mathrm{nm}$ due to the localized surface plasmon resonance effect of AgNPs. (c and d) SEM images showing the morphologies of (c) PAN (scale bar, $2 \mu \mathrm{m}$ ) and (d) PANSion (scale bar, $5 \mu \mathrm{m}$ ) films. Dashed line in (d) indicates the edge between a surface (right) and fracture cross-section (left). (e) Through-thickness elemental distribution of PANSion based on EDS linescan profiles. (f) TEM image of a AgNP embedded PANSion matrix (scale bar, $5 \mathrm{~nm}$ ). Inset shows a histogram of AgNP size (i.e., diameter) distribution (averaged at $\sim 10 \mathrm{~nm}$ ). (g) Current mapping results at various $\phi$ values, by conductive atomic force microscopy (C-AFM, scale bar, $200 \mathrm{~nm}$ ), showing the evolution from an insulative status $(\phi=0)$ to a conductive percolation network ( $\phi=0.5$ to 2.0 ) because of in-situ-grown AgNPs. 
a
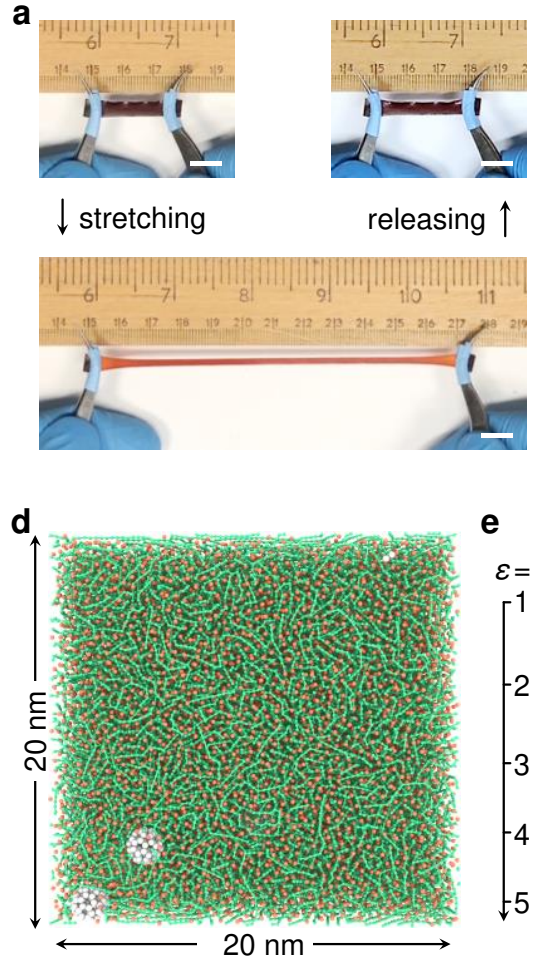

g

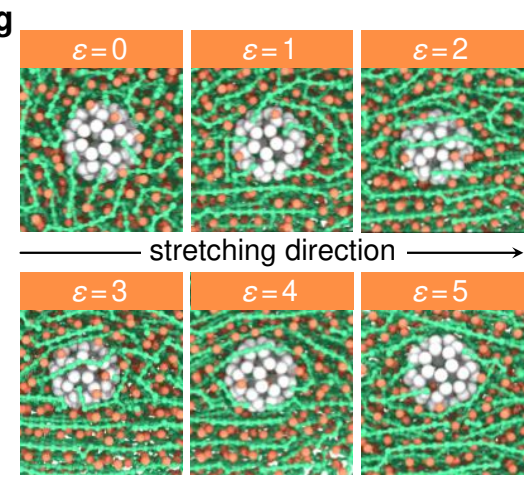

b

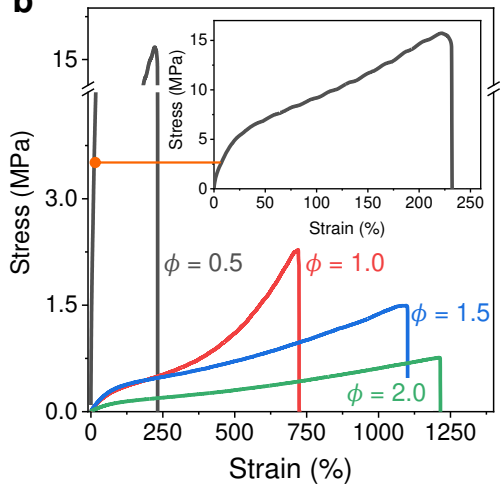

e $\quad$ - stretching direction $\rightarrow$

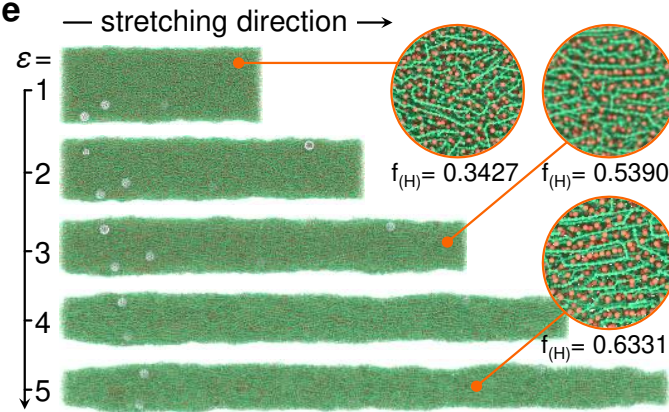

h

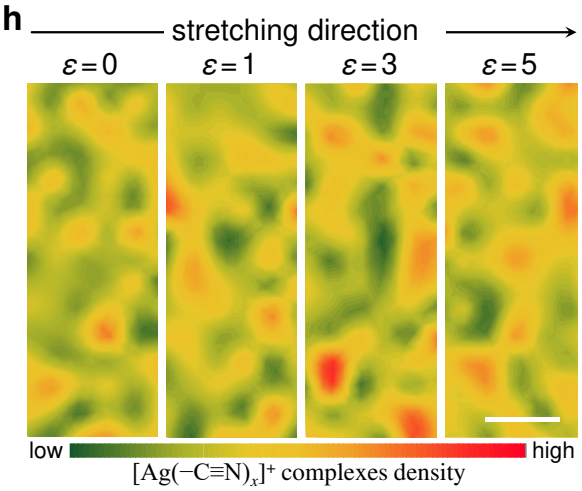

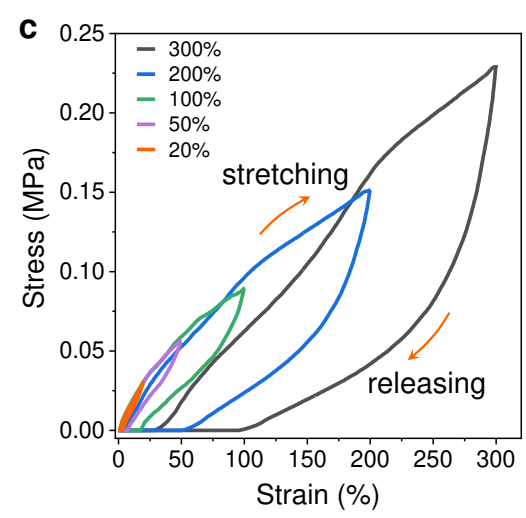

f

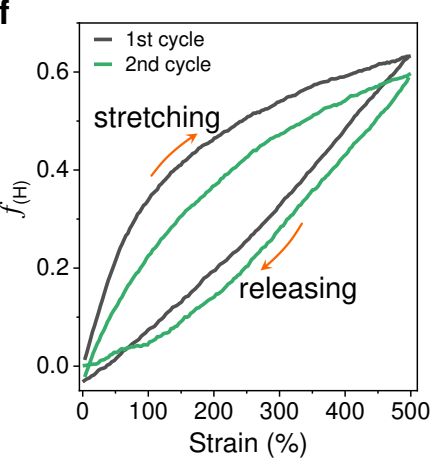

i

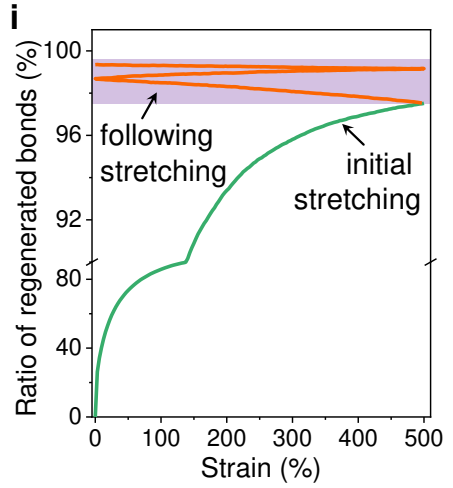

Fig. 3 Mechanical properties of PANSion film. (a) Images showing the high stretchability and recovery capability. Scale bars, $1 \mathrm{~cm}$. (b) Stress-strain curves for $\phi=0.5$ to 2.0. (c) Cyclic stretching-releasing from $20 \%$ to $300 \%$. (d) Constructed model of PANSion film via CG-MD simulation with a dimension of $20 \times 20 \times 20 \mathrm{~nm}^{3}$. Green, dark orange, and light grey represent PAN chains, $\mathrm{Ag}^{+}$, and AgNPs, respectively. (e) Snapshots of network evolutions at various stretches $(\varepsilon)$. Insets show the alignment effect of the PANSion chain at $\varepsilon=1,3$, and 5. (f) Herman's orientation factor $\left(f_{(\mathrm{H})}\right)$ as a function of strain (in \%) during two stretching-releasing cycles. (g) Strong interactions between AgNP and its surrounding PANSion matrix showing no delamination during stretching. (h and i) Density distribution of $[\mathrm{Ag}(-\mathrm{C} \equiv \mathrm{N}) \mathrm{x}]+$ complexes $(\mathrm{h}$, scale bar, $1 \mathrm{~nm})$ and the ratio of regenerated complex bonds (i) under stretching, indicating the dynamic process of dissociation-reformation. 

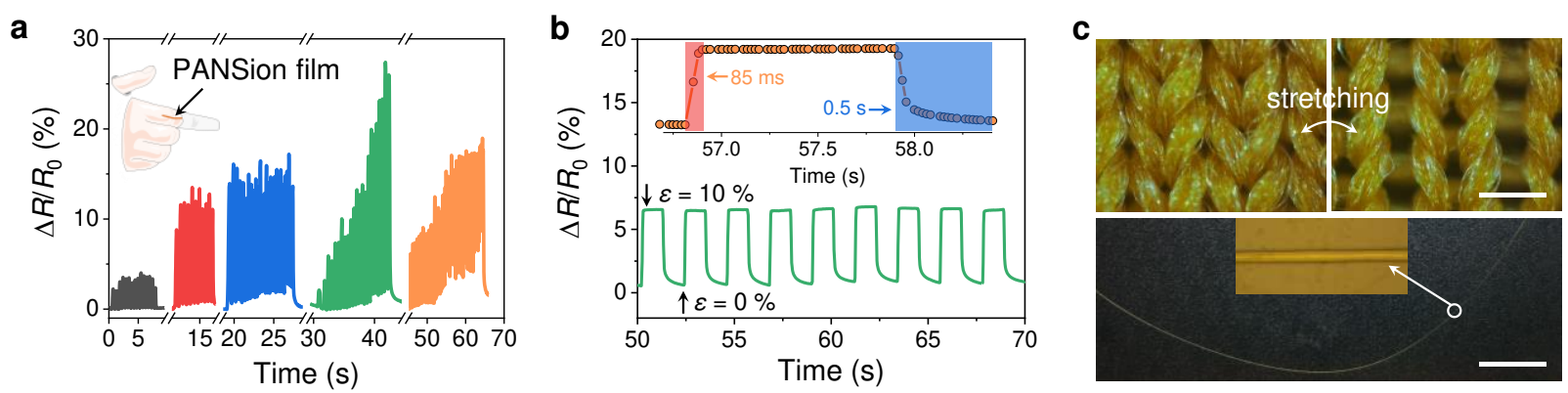

d
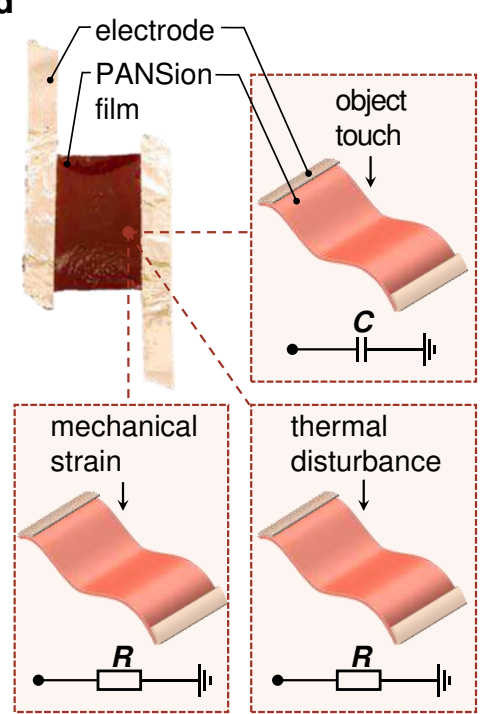

i

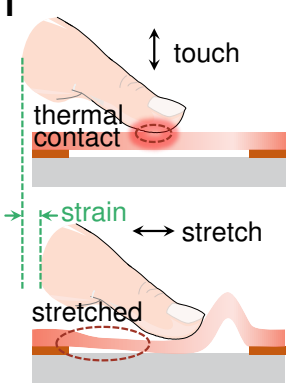

e

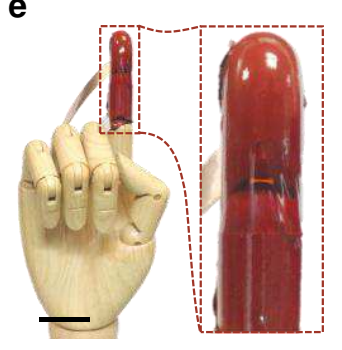

g

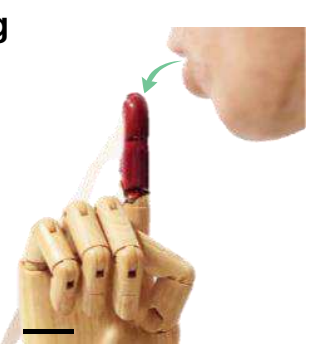

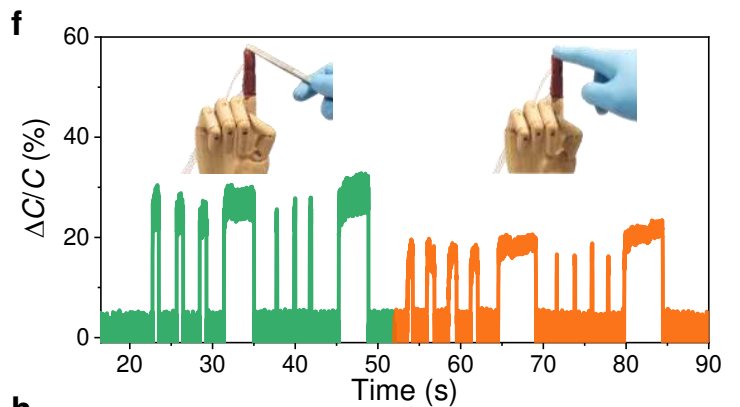

h

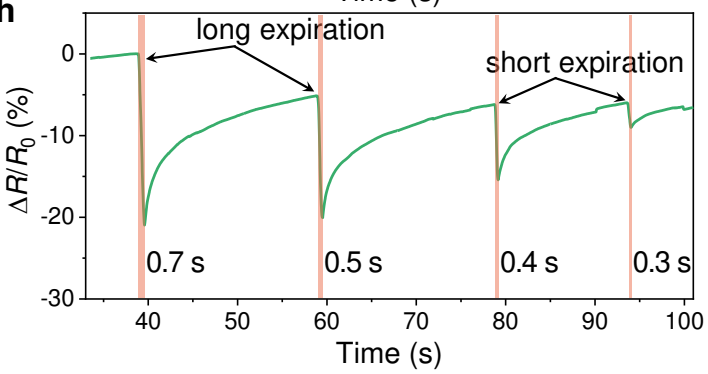

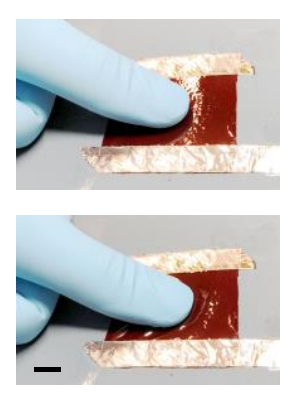

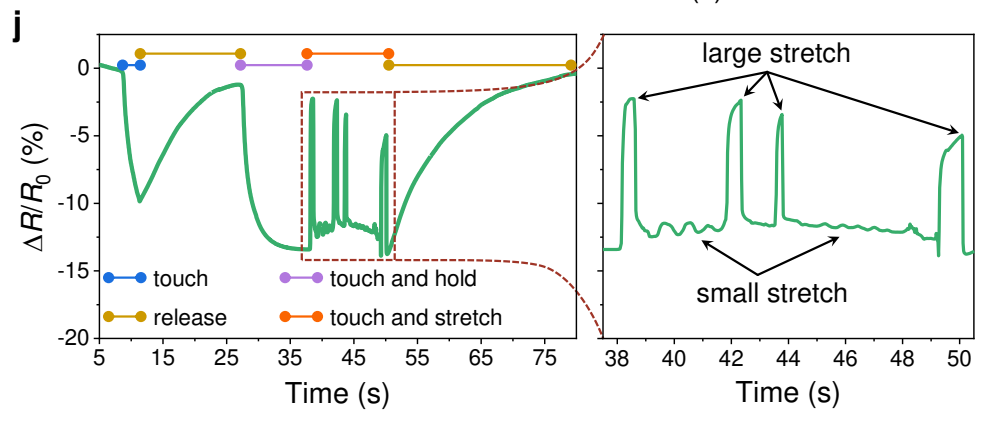

Fig. 4 | Multimodal sensing of a single-layer PANSion electronic skin. (a) Finger flexion recognition of both small-to-large angles, slow-to-fast frequencies, and continuous bending variations. (b) Response and recovery time of PANSion film under a $10 \%$ cyclic strain. (c) Demonstrations on PANSion versatility, including solutions for dip-coating on textiles (top, scale bar, $500 \mu \mathrm{m}$ ) and direct-spun fiber as a functional unit for sensing fabrics (bottom, scale bar, 10 $\mathrm{cm}$ ). (d) Schematic of PANSion-based multimodal sensor for various stimuli sensing, including gentle touch, strain, and temperature. $(\mathbf{e}-\mathbf{h})$ A prosthetic finger wrapped with PANSion film (e, scale bar, $4 \mathrm{~cm}$ ) and its responding signals to touches from a metal bar or human finger (f), and its capability for breathing detection (g, scale bar, $3 \mathrm{~cm}$ ), including long and short expirations (h). (i) Schematic (left) and images (right) showing the human finger touch and stretch on PANSion "skin". Scale bar, $1 \mathrm{~cm}$. (j) Real-time monitoring signals of the touch-stretch actions by a human 
638 finger (temperature and strain stimuli). Enlarged area shows the stretch of both large and small 639 mechanical disturbances. 
a

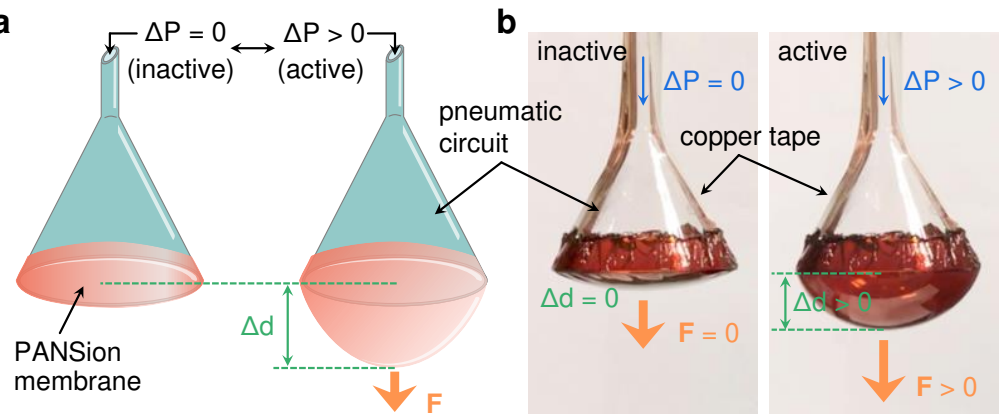

d

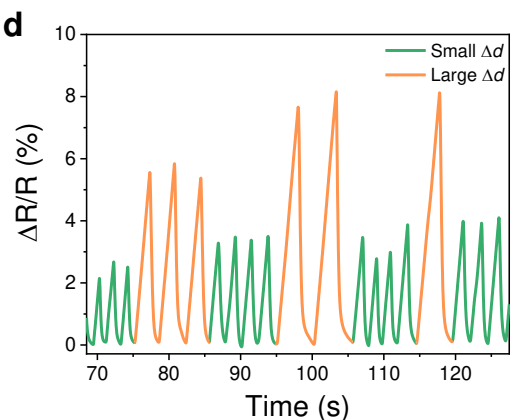

e

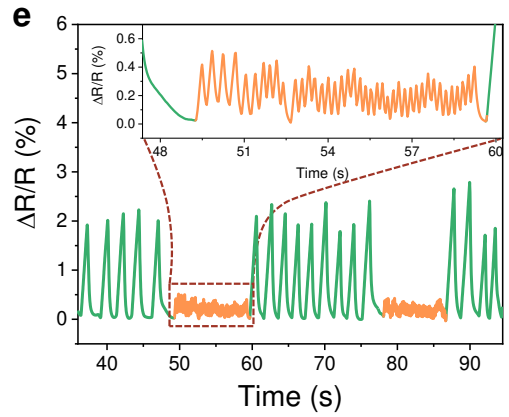

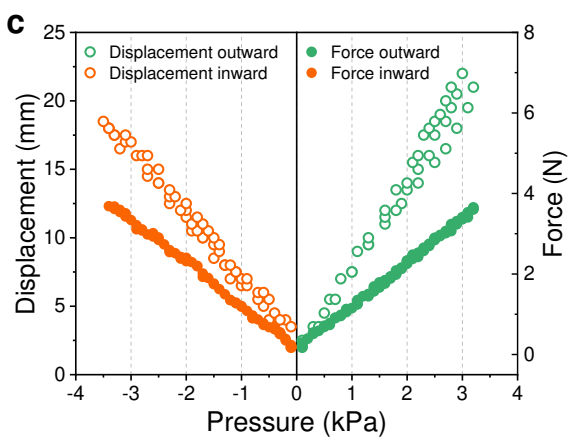

f

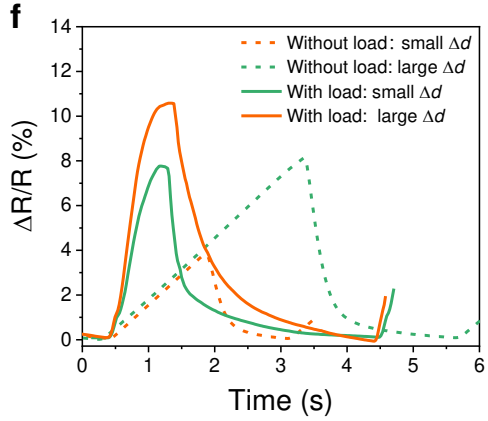

g

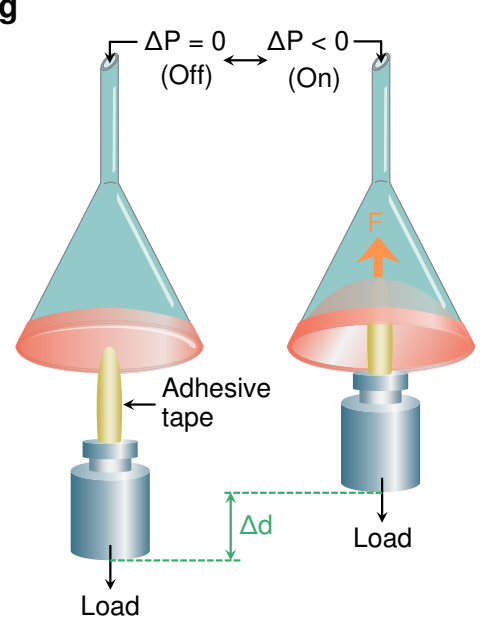

k Object at various temperature

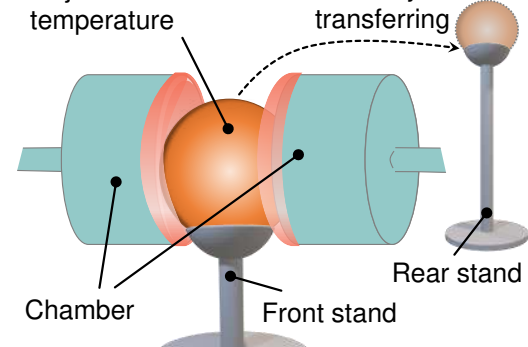

h
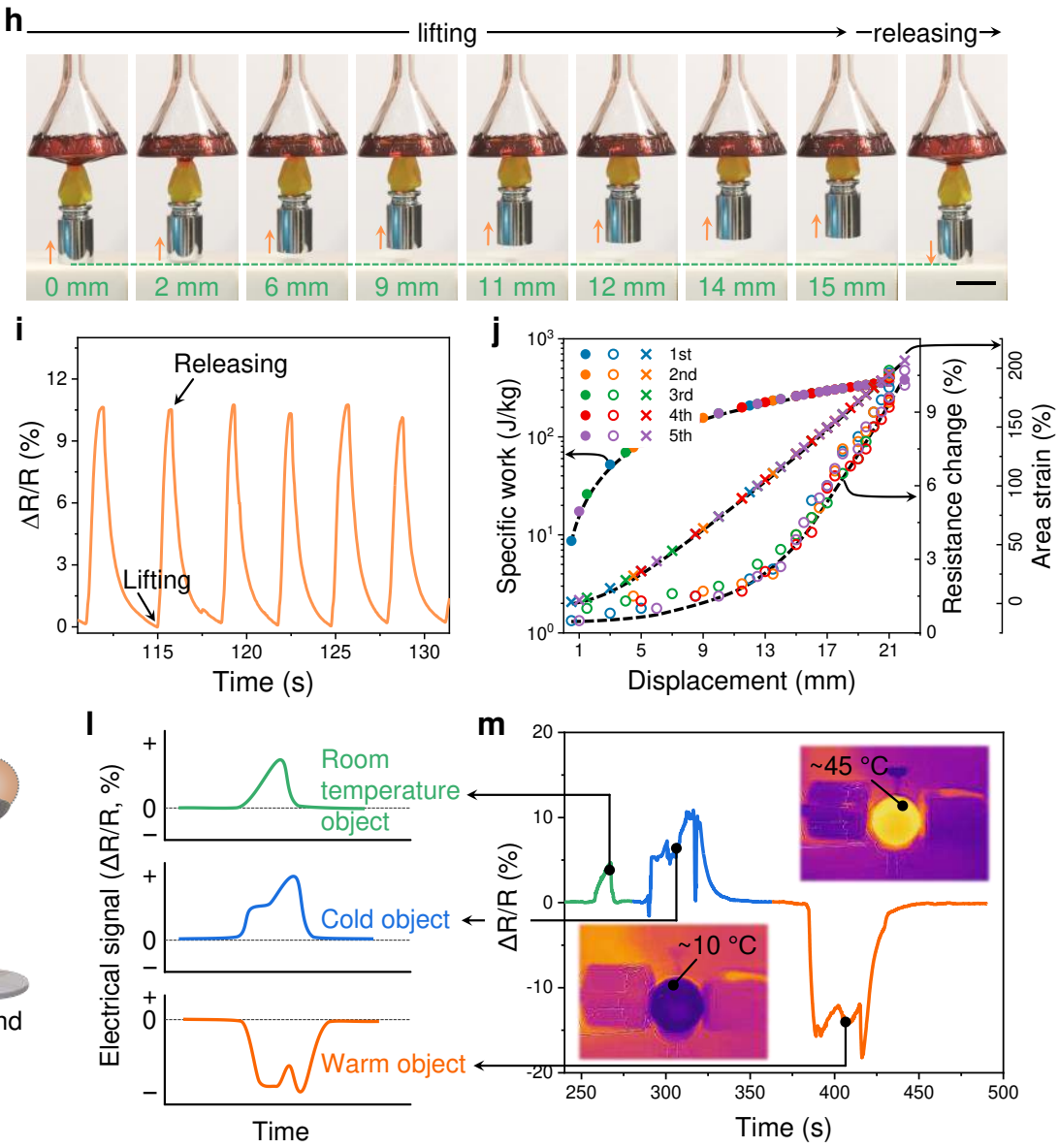

641 Fig. 5 | Self-sensing PANSion actuator. (a) Schematic of the pneumatic actuator using a 642 PANSion film and a cone-shaped holder. (b) Pressure changes within the actuator's chamber 
643 resulting in the PANSion film deformation with a displacement $(\Delta d)$ and output force $(F)$. (c) The 644 displacement and output force as a function of applied pressure, reaching the maximum (inward 645 and outward) of $\sim 18-21 \mathrm{~mm}(\sim 140 \%$ areal strain $)$ and $\sim 3.8 \mathrm{~N}$. (d and e) Sensing signals from the 646 PANSion actuator when performing both (d) small/large deformations (i.e., amplitude) and (e) 647 slow/fast actuation (i.e., frequency). (f) Sensing signal comparison of actuation with and without 648 loads at both small and large displacements. (g) Schematic of using self-sensing PANSion actuator 649 as an artificial muscle. (h) Images showing a lifting-releasing cycle of a 50-g weight. Scale bar, $6502 \mathrm{~cm}$. (i) Real-time sensing signals from the artificial muscle, implying the actuation procedure and 651 behaviors. (j) Output specific work, resistance change, and areal strain while performing a weight652 lifting task at different displacements. (k-m) PANSion-actuator-based soft gripper for object 653 manipulation $(\mathrm{k})$ and the corresponding electrical signals when transferring objects of different 654 temperatures ( 1 and $\mathrm{m})$. 


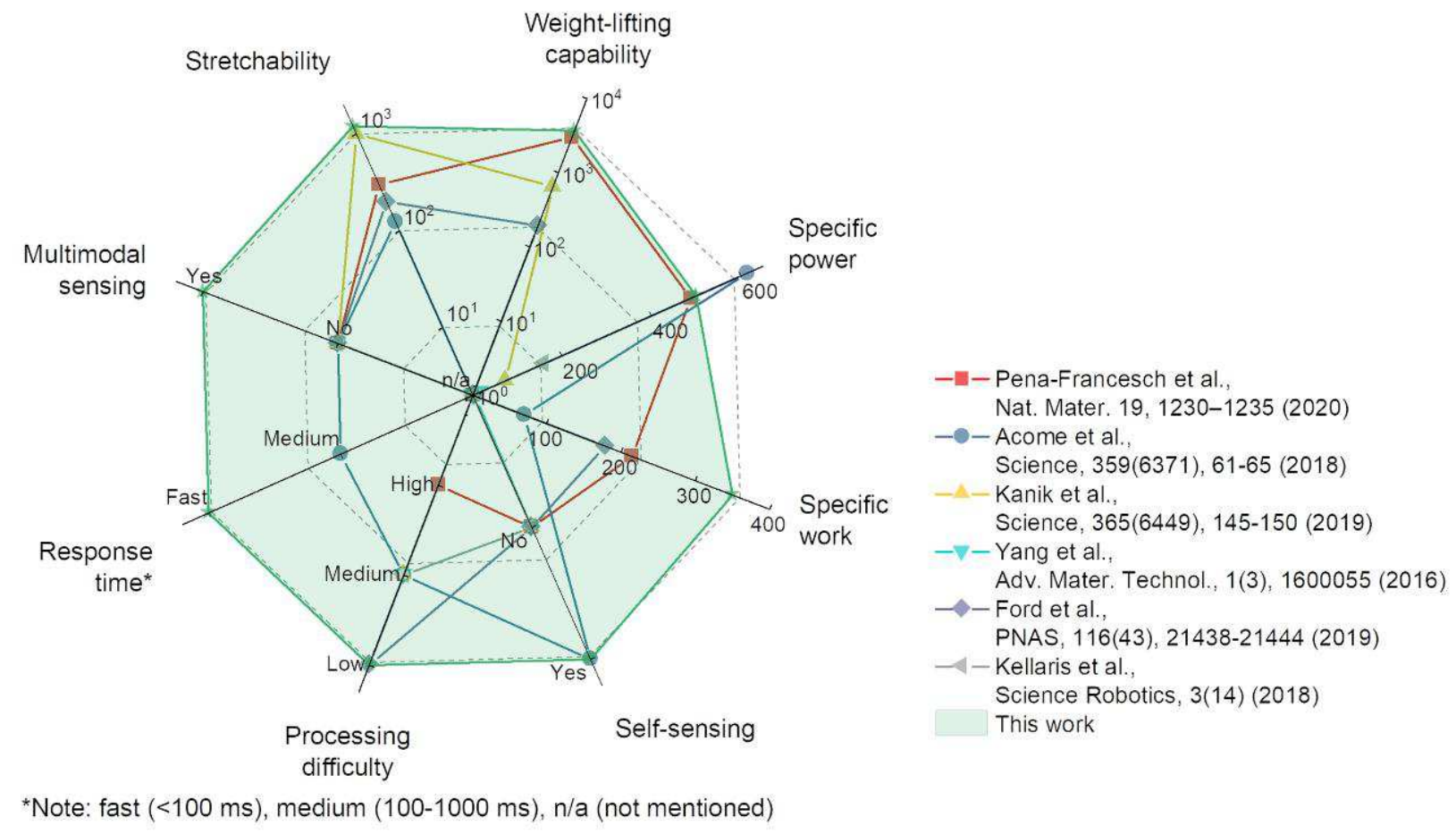

656 Extended Data Fig. 1 | Comprehensiveness of PANSion in properties of sensing and 657 actuation. Radar plot of the performance matrix of PANSion film comparing to other engineering soft actuators in terms of sensing and actuation properties. 
a

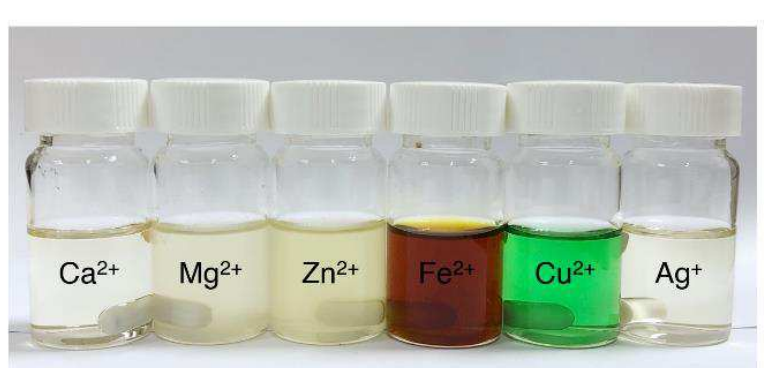

C

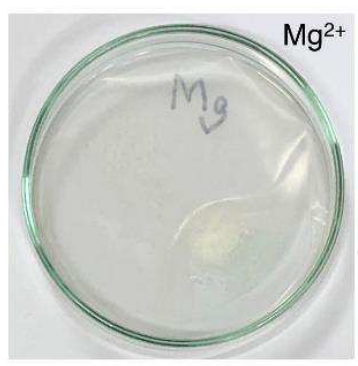

e

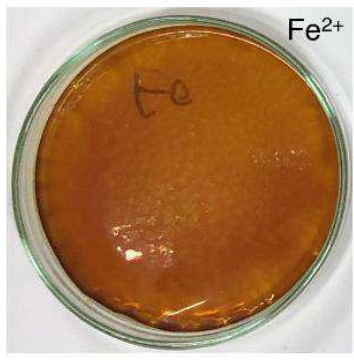

g
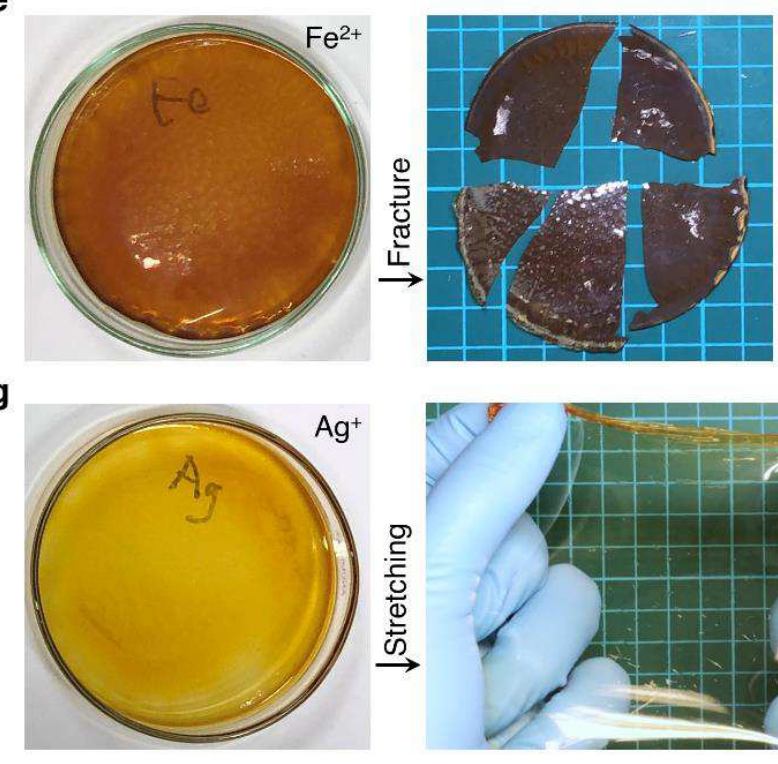

b

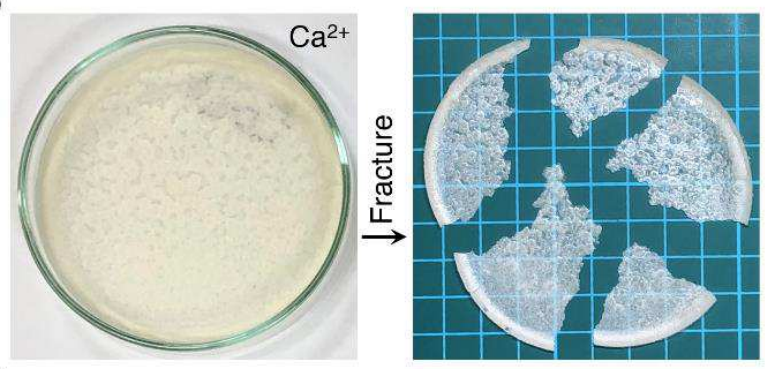

d

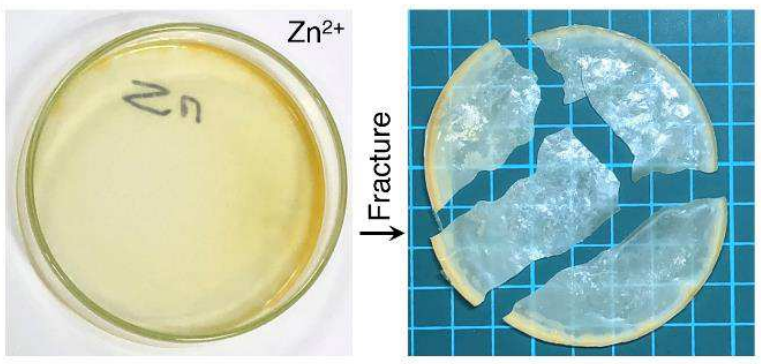

f

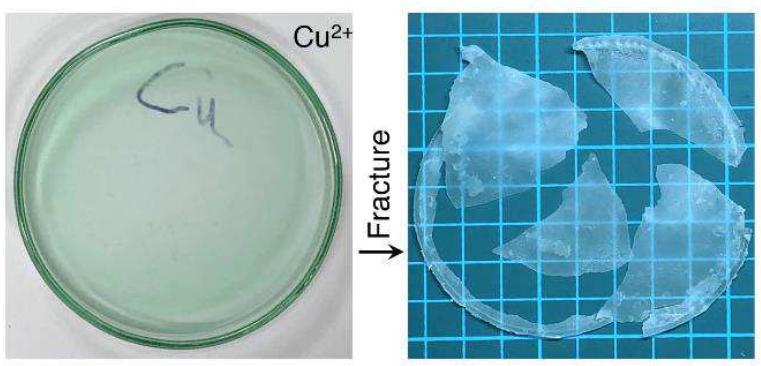

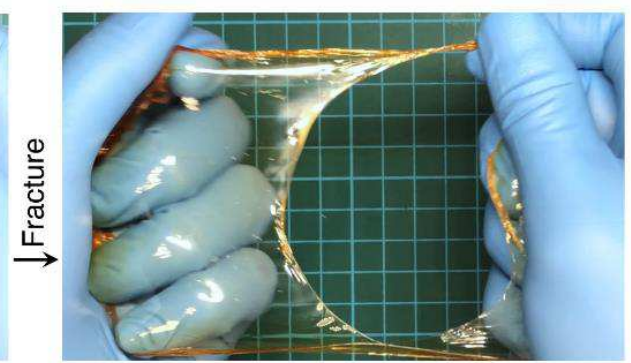

Extended Data Fig. 2 | PAN/metal ion composite films. a, PAN solutions (in DMF) with six 661 different metal ions including calcium $\left(\mathrm{Ca}^{2+}\right)$, magnesium $\left(\mathrm{Mg}^{2+}\right)$, zinc $\left(\mathrm{Zn}^{2+}\right)$, iron $\left(\mathrm{Fe}^{2+}\right)$, copper $662\left(\mathrm{Cu}^{2+}\right)$ and silver $\left(\mathrm{Ag}^{+}\right)$. b-g, PAN/metal ion composite film in a petri dish (left) and the 663 corresponding fracture morphology. All composite films are brittle and easily broken (b-f) except 664 the one with silver ion (g). PAN/silver ion composite film (namely, PANSion film) is soft and 665 stretchable. 

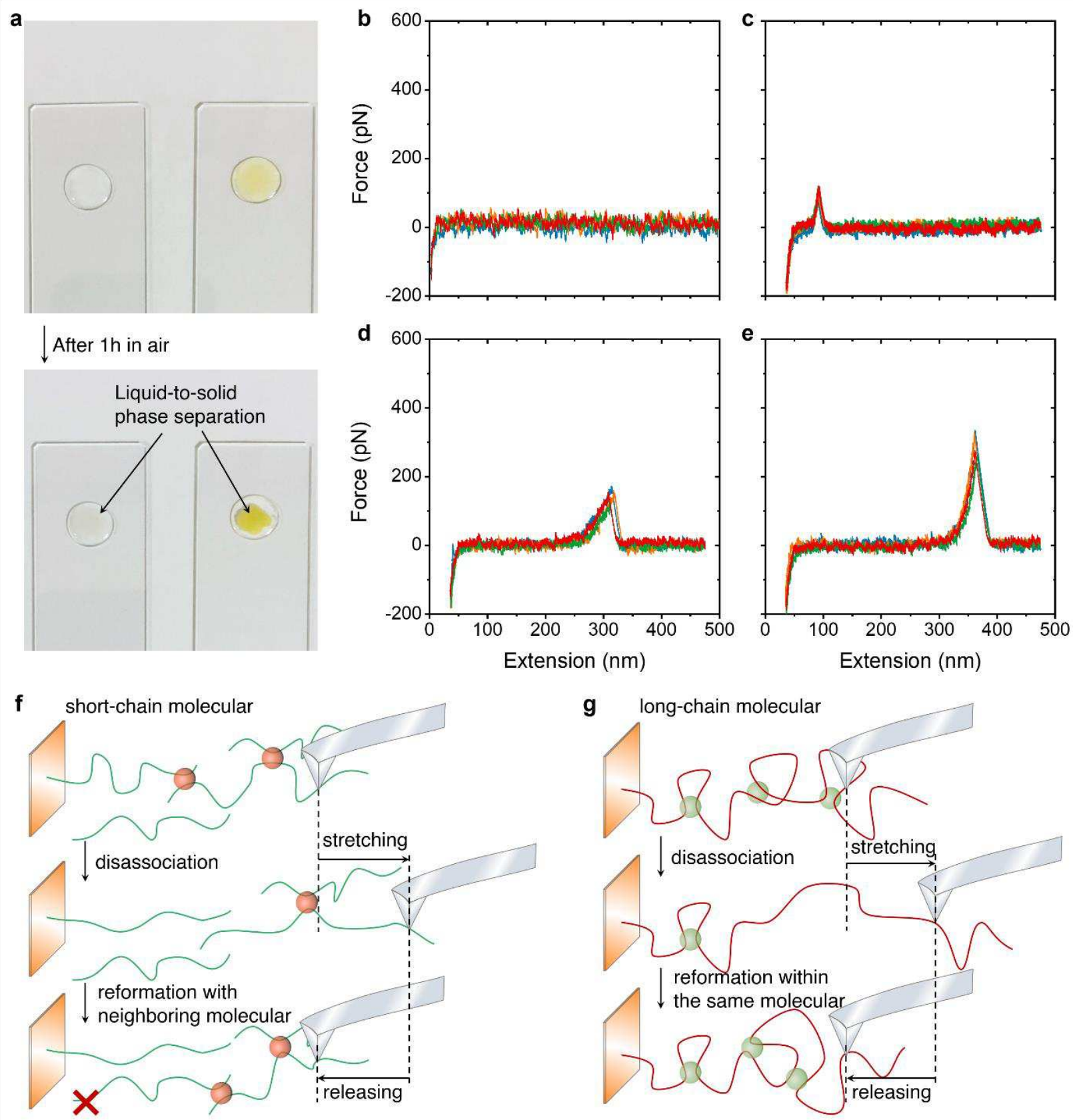

Extended Data Fig. $3 \mid$ Mechanical properties of $\left[\operatorname{Ag}(-\mathrm{C} \equiv \mathrm{N})_{x}\right]^{+}$characterized by AFM-SMFS. a, Phase separation of PAN and PANSion solutions after an air exposure. $\mathbf{b}-\mathbf{e}$, Representative force-extension curves of PAN and PANSion chains. $\mathbf{f}$ and $\mathbf{g}$, Schematic of the stretching-releasing process via AFM-SMFS for (f) short-chain molecule and (g) long-chain molecule. 

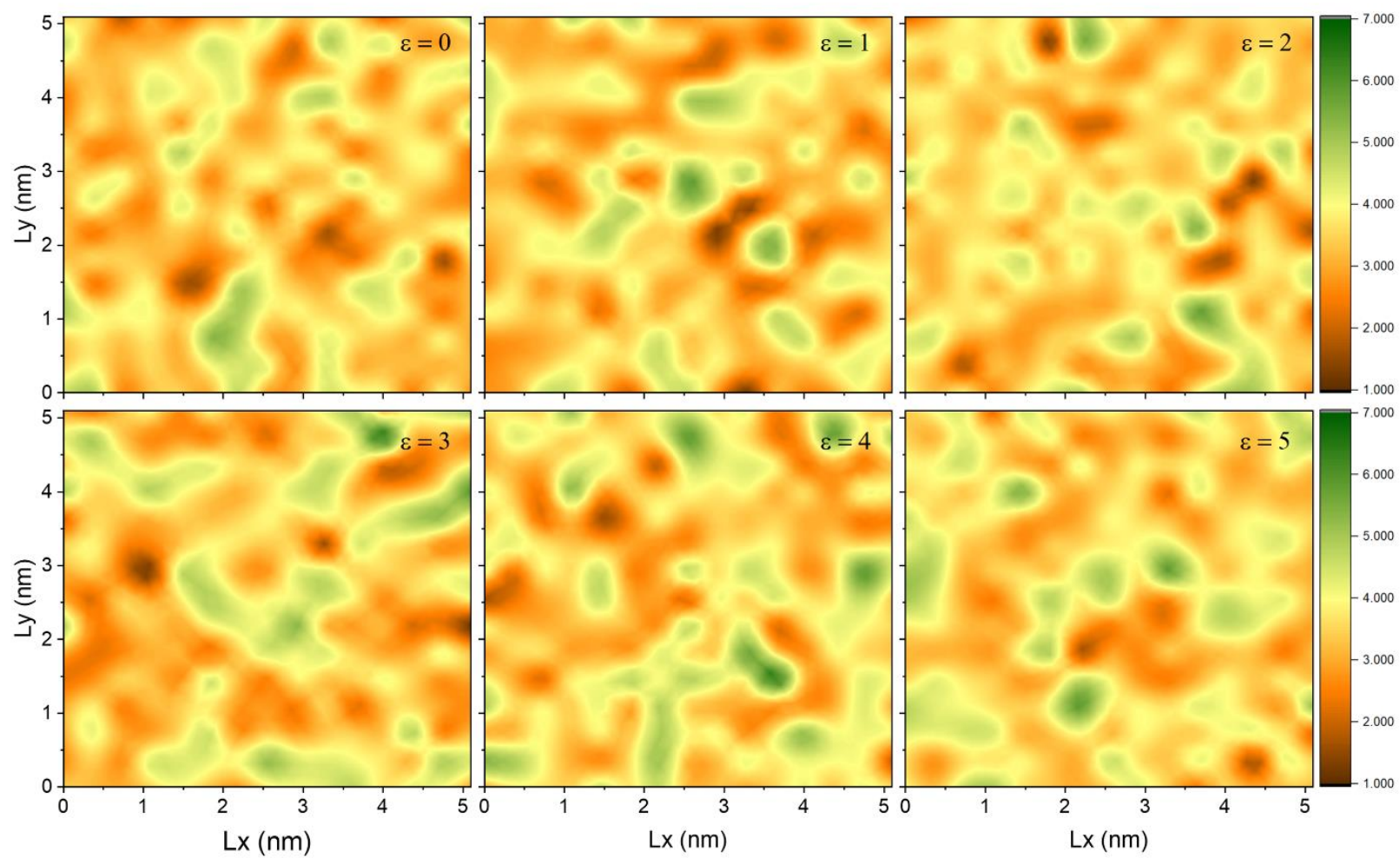

Extended Data Fig. 4 | Mappings of the number of coordination bonds of PANSion film under stretching. No obvious difference in the total coordination bonds was observed when the strain was increased from 0 to 5 . 
a

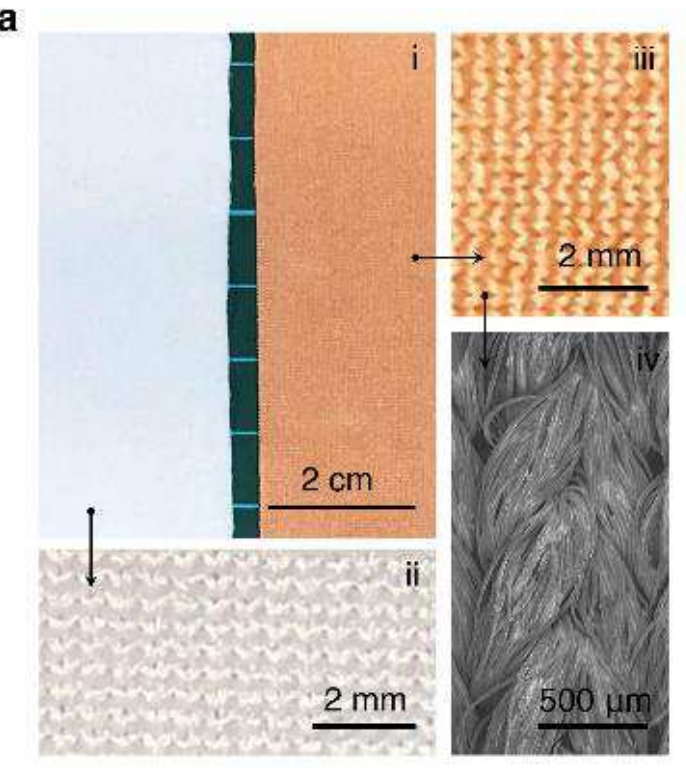

b
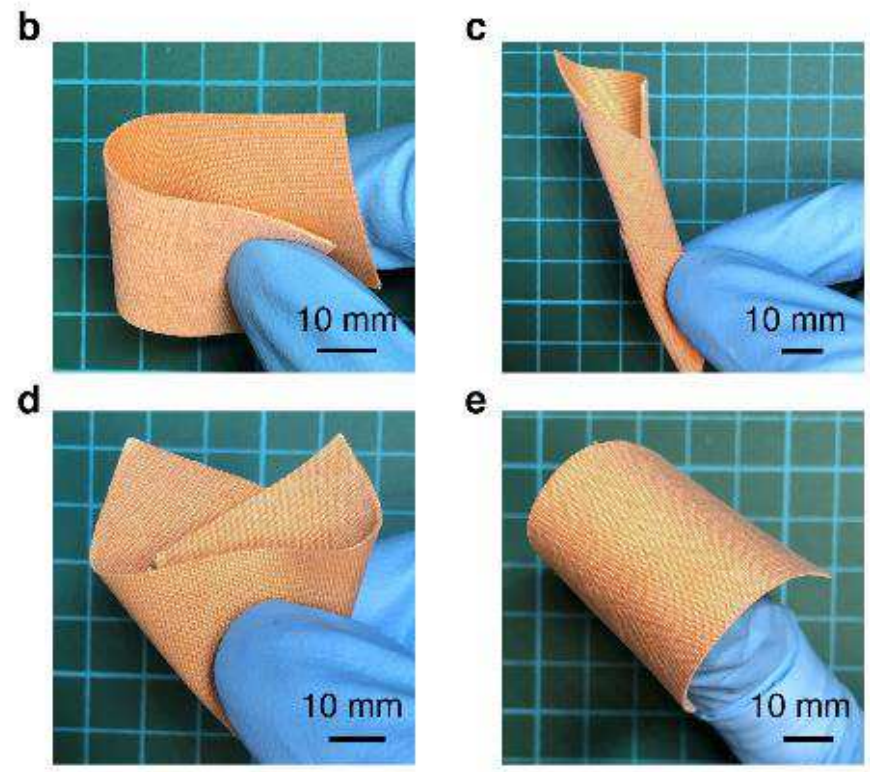

e

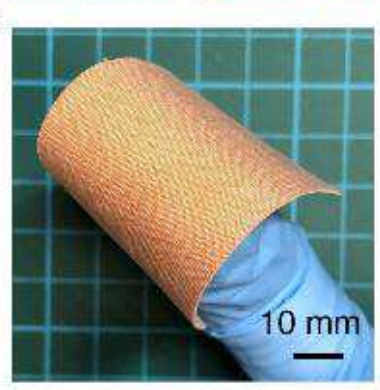

f 200

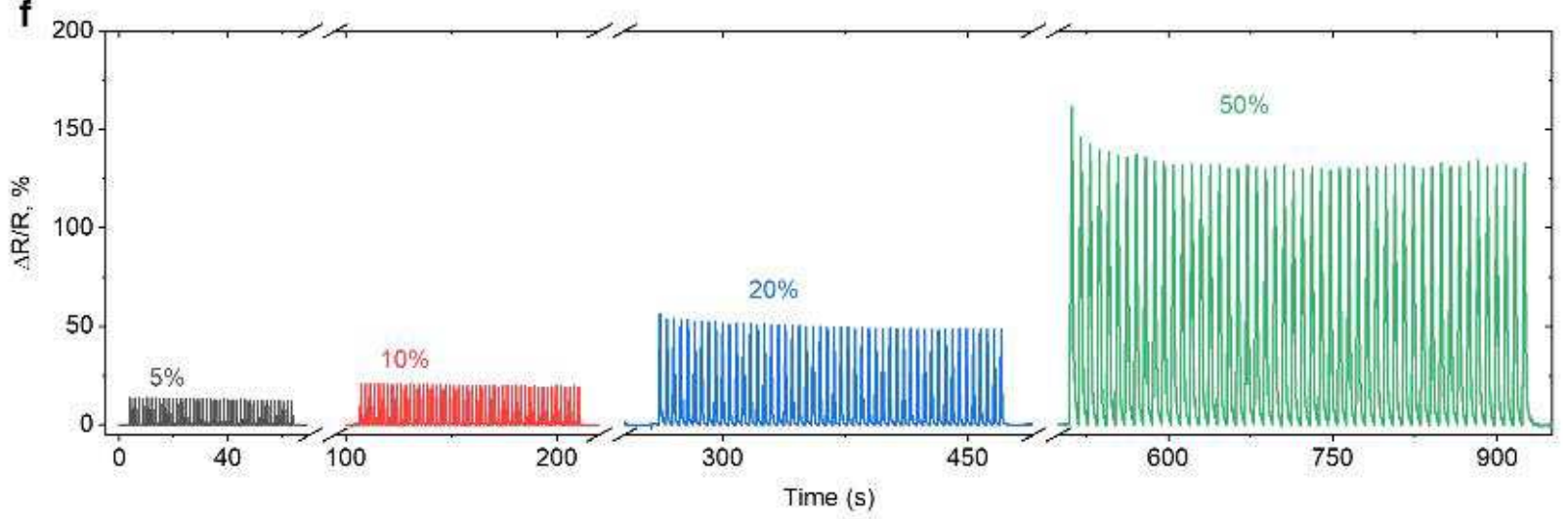

Extended Data Fig. 5 | PANSion solution coated PU fabric. a, A PU fabric before and after PANSion solution coating: (i) original PU fabric (left) and the one after coating (right); (ii and iii) enlarged area showing the knitting structure before (ii) and after (iii) coating; (iv) SEM image of the coated fabric, showing the yarn structure without any aggregation of PANSion polymer. b, The knitted PU fabric after PANSion solution coating is still as flexible as the uncoated one, demonstrating no changes to the mechanical properties of the original fabric. $\mathbf{c}-\mathbf{e}$, Good wearing properties are still retained, including twistability, foldability, and drapability. f, Sensing property of the coated PU fabric at strain from 5 to $50 \%$. 
a
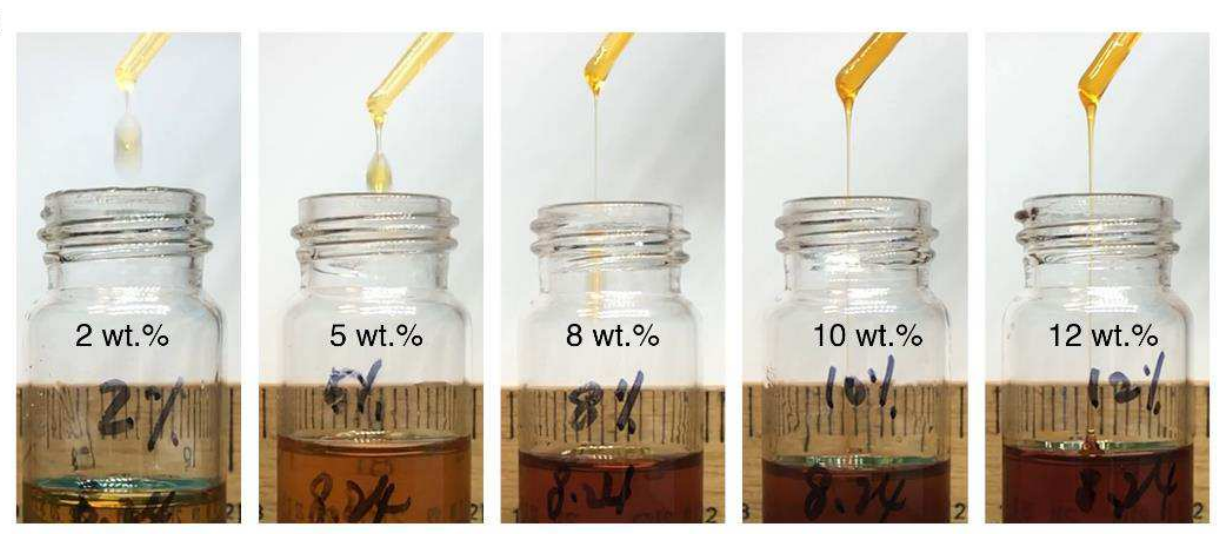

b

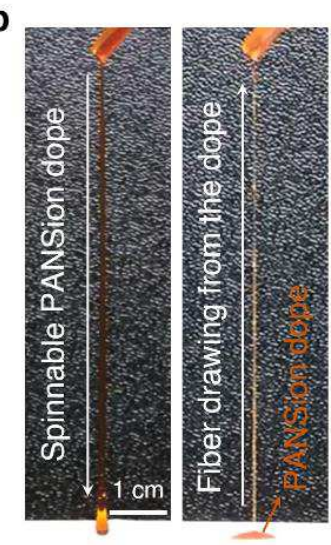

C

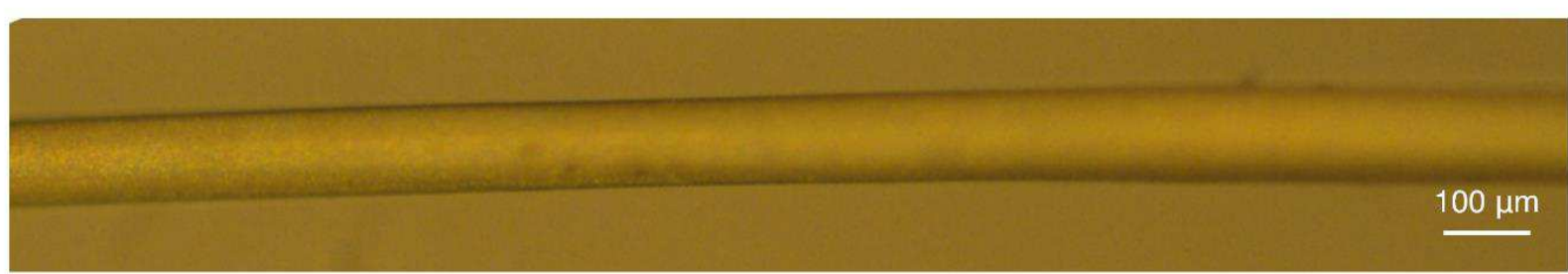

d

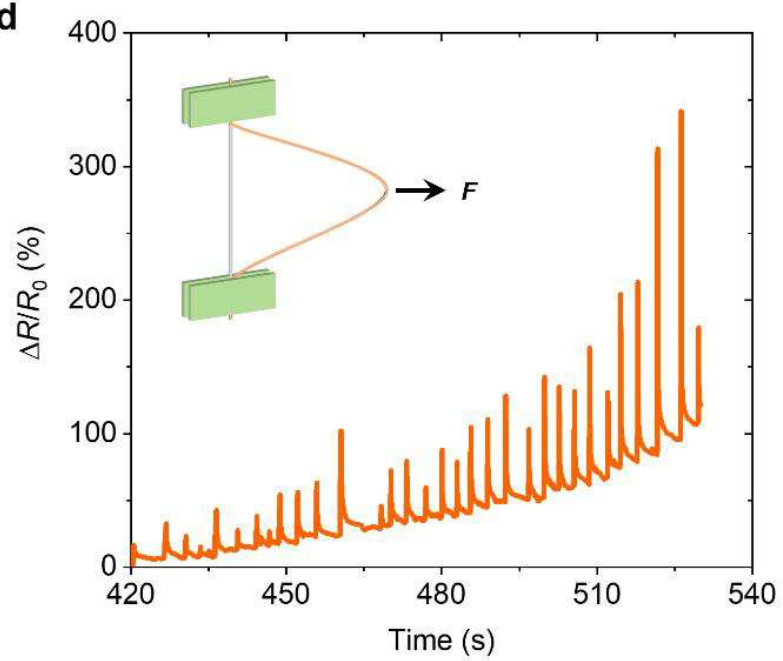

e

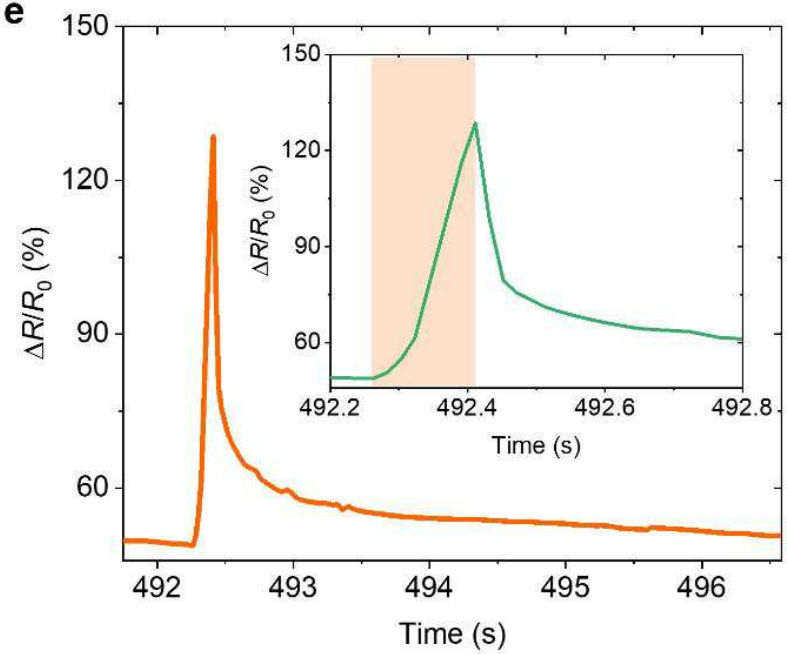

684

Extended Data Fig. 6 | PANSion fiber spinning and fiber's sensing property. a, The spinnability of PANSion solution $(\phi=2.0)$ with a different base concentration of PAN. After storing PANSion solutions at room temperature for several days, the viscosity of solutions is different from each other. $10 \mathrm{wt} \%$ and $12 \mathrm{wt} \%$ PANSion solutions possess suitable viscoelastic properties for direction fiber spinning, as demonstrated by the continuous dope dripping from a plastic pipette. $\mathbf{b}$, The dripping test of $10 \mathrm{wt} \%$ PANSion solution from a height of $\sim 8 \mathrm{~cm}$ (left) shows good flowability and spinnability. A single fiber can be directly drawn from a PANSion dope in the air without any post-treatment. c, Optical image of a PANSion fiber with a diameter $\sim 90 \mu \mathrm{m}$. $\mathbf{d}$ and $\mathbf{e}$, Sensing property of PANSion fibers. The as-spun PANSion fiber is as stretchable as the PANSion film. Interestingly, the electromechanical sensing property is also retained. It displays distinguishable resistance change responses for various external strains (d) with decent response time (e). 
a

697

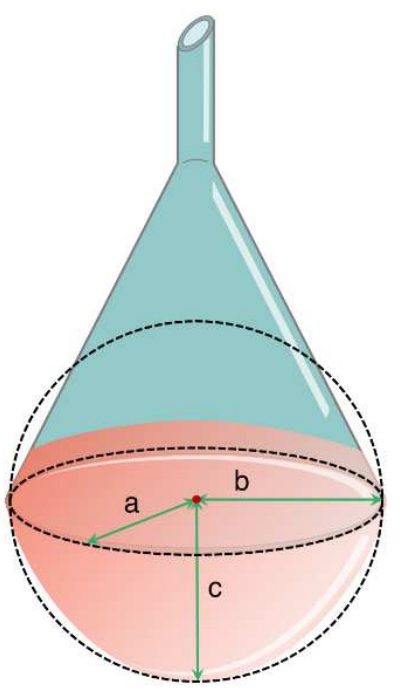

b

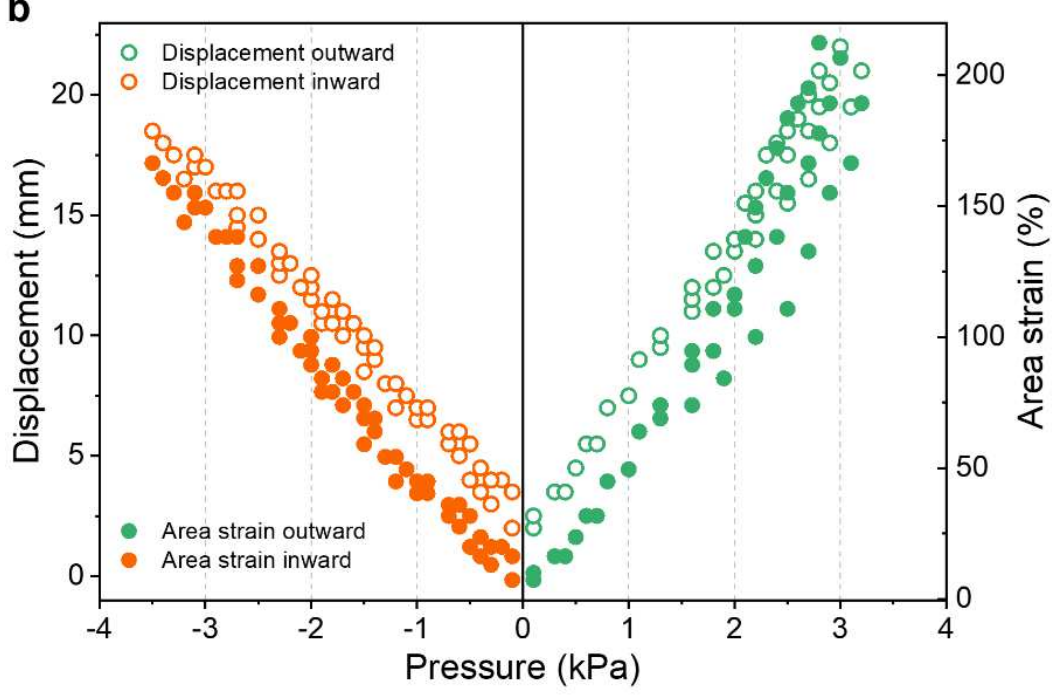

698

699

700

Extended Data Fig. 7 | Displacement $(\mathrm{mm})$ and area strain (\%) as a function of applied pressure. a, Schematic of how the area strain was calculated (supplementary text S7). b, Results of displacement and area strain when pressure was changed accordingly. 

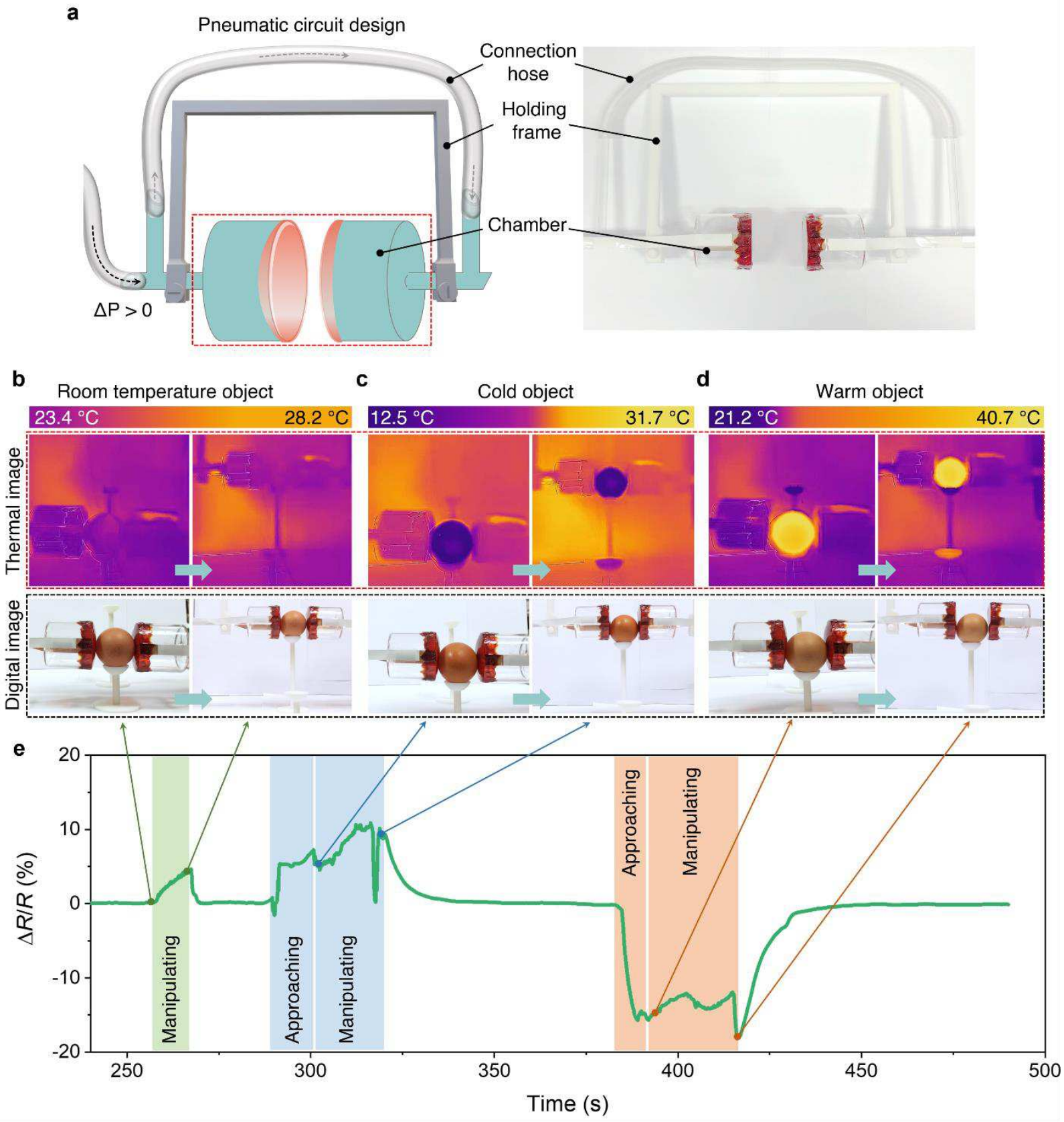
for object manipulation. a, The schematic of the pneumatic circuit design for a soft gripper based on a self-sensing PANSion actuator (left) and the digital image of the gripper (right). b-d, The thermal and digital images showing that the self-sensing actuator-based gripper is transferring various objects: room temperature object (b), cold object (c), and warm object (d). e, The corresponding real-time electrical signals from the gripper can be further used as input for smart robotics to distinguish the thermal profile of the transferred object. 


\section{Supplementary Files}

This is a list of supplementary files associated with this preprint. Click to download.

- Video5.mp4

- Supplementarylnformation.pdf

- Video1.mp4

- Video6.mp4

- Video4.mp4

- Video2.mp4

- Video3.mp4 